\title{
Well-balanced finite volume schemes for pollutant transport on unstructured meshes
}

\author{
Fayssal Benkhaldoun*† $\quad$ Imad Elmahi $\ddagger \quad$ Mohammed Seaïd ${ }^{\S}$
}

\begin{abstract}
Pollutant transport by shallow water flows on nonflat topography is presented and numerically solved using a finite volume scheme. The method uses unstructured meshes, incorporates upwinded numerical fluxes and slope limiters to provide sharp resolution of steep bathymetric gradients that may form in the approximate solution. The scheme is non-oscillatory and possesses conservation property that conserves the pollutant mass during the transport process. Numerical results are presented for three test examples which demonstrate the accuracy and robustness of the scheme and its applicability in predicting pollutant transport by shallow water flows. In this paper, we also apply the developed scheme for a pollutant transport in the strait of Gibraltar. The scheme is efficient, robust and may be used for practical pollutant transport phenomena.
\end{abstract}

Keywords. Shallow water equations, Pollutant transport, Finite volume method, SRNH scheme, Unstructured grids, Strait of Gibraltar

\section{Introduction}

During the last years the increase of pollution in rivers, lagoons and coastal regions has attracted much interest in numerical methods for the prediction of its transport and dispersion. In many situations, this pollution problem has detriment impact on the ecology and environment and may cause potential risk on the human health and local economy. Efficient and reliable estimates of damages on the water quality due to pollution could play essential role in establishing control strategy for environmental protection. Introduction and utilization of such measures are impossible without knowledge of various processes such as formation of water flows and transport of pollutants. The mathematical models and computer softwares could be very helpful to understand the dynamics of both, water flow and pollutant transport. In this respect mathematical modeling of water flows and the processes of transport-diffusion of pollutants could play a major role in establishing scientifically justified and practically reasonable programs for long-term measures for a rational use of water resources, reduction of pollutants discharge from particular sources, estimation of the impact in the environment of possible technological improvements, development of methods and monitoring facilities, prediction and quality management of the environment, etc. The success of the mathematical and computer methods in solving practical problems depends on the convenience of the models and the quality of the software used for the simulation of real processes.

\footnotetext{
*LAGA, Université Paris 13, 99 Av J.B. Clement, 93430 Villetaneuse, France (fayssal@math.univ-paris13.fr)

${ }^{\dagger}$ CMLA, ENS, 61 Av. Pdt Wilson, 94235 Cachan Cedex, France (fayssal@cmla.ens-cachan.fr)

${ }^{\ddagger}$ ENSAO Complex Universitaire, B.P. 473, 60000 Oujda, Morocco (ielmahi@ensa.univ-oujda.ac.ma)

${ }^{\S}$ Fachbereich Mathematik, Technische Universität Kaiserslautern, 67663 Kaiserslautern, Germany (seaid@mathematik.uni-kl.de)
} 
Clearly, the process of pollutant transport is determined by the characteristics of the fluid flow and the properties of the pollutant. Thus, dynamics of the fluid and dynamics of the pollutant must be studied using a mathematical model made of two different but dependent model variables: (i) a hydrodynamic variable defining the dynamics of the flow, and (ii) a concentration variable defining the transport of the pollutant. In the current work, the hydrodynamic model is based on a two-dimensional shallow water equations while, an advection-diffusion equation is used for the pollutant transport. For environmental flows, the shallow water system is a suitable model for adequately describing significant hydraulic processes. The different characteristics of pollutants require an appropriate model to describe their dynamics, nevertheless for a wide class of dispersed substances the standard advection-diffusion equation can be used. The interaction between the two processes gives rise to a hyperbolic system of conservation laws with source terms.

The accurate solution of shallow water flows is of major importance in most of pollutant transport predictions. In many practical applications the shallow water equations has to be solved on nonflat and rough beds, and also on topographic structures covering different spatial scales. Thus, the treatment of topography and friction source terms is of major importance in these applications. It is well known that shallow water equations on nonflat topography have steady-state solutions in which the flux gradients are nonzero but exactly balanced by the source terms. This well-balanced concept is also known by exact conservation property (C-property), compare [13, 11, 36]. Computational techniques using finite difference, finite element and finite volume methods have been extensively reported in the literature. Although widely applied to shallow water equations, the finite difference technique has the major drawback that it does not guarantee strict conservation of mass and momentum. Furthermore, the necessity of including process across a range of spatial scales means that techniques capable of operating on unstructured meshes will be more appropriate than those such as the finite difference methods which rely on structured and often regular meshes. The finite element method has been used with irregular meshes of triangular or quadrilateral elements to model shallow water flows $[17,15]$. However, the finite element method can experience difficulty when both subcritical and supercritical flows are encountered [3], and may produce solutions with local mass conservation errors in some implementations [19]. The finite volume method is therefore adopted in the present work. For a comprehensive review of recent developments in finite volume methods for shallow water equations we refer to [35].

Various numerical methods developed for general systems of hyperbolic conservation laws have been applied to the shallow water equations. For instance, most shock-capturing finite volume schemes for shallow water equations are based on approximate Riemann solvers which have been originally designed for hyperbolic systems without accounting for source terms such as bed slopes and friction losses. Therefore, most of these schemes suffer from numerical instability and may produce nonphysical oscillations mainly because dicretizations of the flux and source terms are not well-balanced in their reconstruction. The well-established Roe's scheme [31] has been modified by Bermúdez and Vázquez [11] to treat source terms. This method has been improved by Vázquez [36] for general one-dimensional channel flows. However, for practical applications, this method may become computationally demanding due to its treatment of the source terms. Alcrudo and Garcia-Navarro [5] have presented a Godunov-type scheme for numerical solution of shallow water equations. Alcrudo and Benkhaldoun [4] have developed exact solutions for the Riemann problem at the interface with a sudden variation in the topography. The main idea in their approach was to define the bottom level such that a sudden variation in the topography occurs at the interface of two cells. LeVeque [24] proposed a Riemann solver inside a cell for balancing the source terms and the flux gradients. However, the extension of this scheme for unstructured meshes is not trivial. Numerical methods based on surface gradient techniques have also been applied to shallow water equations by Zhou et al. [39]. The TVD-MacCormak scheme has been used by Ming-Heng [27] to solve water flows in variable bed topography. A different approach based on local hydrostatic reconstructions have been studied by Audusse el al. [7] for open channel flows with topography. 
The performance of discontinuous Galerkin methods has been examined by Xing and Shu [38] for some test examples on shallow water flows. A central-upwind scheme using the surface elevation instead of the water depth has been used by Kurganov and Levy [22]. Vukovic and Sopta [37] extended the ENO and WENO schemes to one-dimensional shallow water equations. On the other hand, numerical methods based on kinetic reconstructions have been studied by Perthame and Simeoni in [30] for one-dimensional problems. In the framework of kinetic schemes, Seaïd [34] proposed a class of relaxation methods for solving shallow water equations.

In this paper, we describe the development of a finite volume non-homogeneous Riemann solver (SRNH) for pollutant transport by shallow water equations. The original SRNH scheme has been recently proposed by Benkhaldoun and Quivy in [9] and analyzed by Sahmim and Benkhaldoun in [33]. The SRNH scheme has also been numerically examined by Sahmim et al. [32] for the one-dimensional shallow water equations. Typically, in the above mentioned references, the scheme has been implemented for structured meshes. However, unstructured meshes can be highly advantageous based on their ability to provide local mesh refinement near important bathymetric features and structures. As a consequence, the ability to provide local mesh refinement where it needed leads to improved accuracy for a given computational cost as compared to methods that use structured meshes. Therefore, our objective in the current study, is to extend the SRNH scheme for the numerical simulation of two-dimensional pollutant transport problems on unstructured meshes. The main advantages of the proposed SRNH method are (i) the implementation on unstructured meshes allowing for local mesh refinement during the simulation process, (ii) the simultaneous advection in time of the water flow and the pollutant concentration, solving both problems at the same time and with the same accuracy (ii) the ability to handle calculations of slowly varying flows or concentrations as well as rapidly varying flows containing also shocks or discontinuities, and (iii) the capability to satisfy the exact C-property and to guarantee positive values of both, water level and pollutant concentration in the transient simulations. In the computations presented in this paper we have used residual in the concentration of pollutants as a monitoring function for mesh refinements. Results presented in this paper demonstrate high resolution of the proposed method and confirm its capability to provide accurate and efficient simulations for pollutant transport by shallow water flows including complex topography and friction forces on unstructured grids.

This paper is organized as follows. In section 2 we present the mathematical equations used to model pollutant transport problems. Formulation of the SRNH scheme is discussed in section 3. Computational results are illustrated in section 4 for two hypothetical examples in smooth rectangular domains. We finally present an application of the SRNH scheme to a pollutant transport event in the strait of Gibraltar. Section 5 summarizes the paper with some concluding remarks.

\section{Two-Dimensional Pollutant Transport Equations}

Shallow water equations have been widely used to model free surface flows of a fluid under the influence of gravity. This class of equations uses the assumption that the vertical scale is much smaller than any typical horizontal scale and can be derived from the depth-averaged incompressible Navier-Stokes equations. For two-dimensional flow problems, these equations are

$$
\begin{aligned}
\partial_{t} h+\partial_{x}(h u)+\partial_{y}(h v) & =0, \\
\partial_{t}(h u)+\partial_{x}\left(h u^{2}+\frac{1}{2} g h^{2}\right)+\partial_{y}(h u v) & =-g h\left(S_{0 x}-S_{f x}\right), \\
\partial_{t}(h v)+\partial_{x}(h u v)+\partial_{y}\left(h v^{2}+\frac{1}{2} g h^{2}\right) & =-g h\left(S_{0 y}-S_{f y}\right),
\end{aligned}
$$


where $g$ is the gravitational acceleration, $h(t, x, y)$ is the water depth, $u(t, x, y)$ and $v(t, x, y)$ are the depth-averaged velocities in the $x$ - and $y$-direction, respectively. The source terms in (2.1) account for various physical and geometric effects. Here, $S_{0 x}$ and $S_{0 y}$ are the bed slopes given by

$$
S_{0 x}=\partial_{x} Z, \quad S_{0 y}=\partial_{y} Z
$$

with $Z(x, y)$ denotes the bottom topography, while $S_{f x}$ and $S_{f y}$ are the friction losses along the $x$ and $y$-direction, and are defined by

$$
S_{f x}=\eta^{2} \frac{u \sqrt{u^{2}+v^{2}}}{h^{4 / 3}}, \quad S_{f y}=\eta^{2} \frac{v \sqrt{u^{2}+v^{2}}}{h^{4 / 3}}
$$

where $\eta$ is the Manning roughness coefficient. It well known that the system (2.1) is strictly hyperbolic with real and distinct eigenvalues given by

$$
\begin{array}{lll}
\lambda_{1}=u-\sqrt{g h}, & \lambda_{2}=u, & \lambda_{3}=u+\sqrt{g h}, \\
\mu_{1}=v-\sqrt{g h}, & \mu_{2}=v, & \mu_{3}=v+\sqrt{g h} .
\end{array}
$$

The transport of a pollutant is modelled by the standard advection-diffusion equation

$$
\partial_{t}(h C)+\partial_{x}(h u C)+\partial_{y}(h v C)=\nabla \cdot(\mathbf{D} \nabla(h C))+h Q,
$$

where $C(t, x, y)$ is the depth-averaged pollution concentration, $Q$ is the depth-averaged pollutant source or sink, and $\mathbf{D}$ is a $2 \times 2$ diffusion matrix. In practical situations the diffusion coefficients depend on water depth, flow velocity, bottom roughness, wind and vertical turbulence, compare [25] for more details. For the purpose of the present work, the problem of the evaluation of diffusion coefficients is not considered.

For simplicity in presentation we rewrite the equations (2.1) and (2.5) in a conservative form as

$$
\partial_{t} \mathbf{W}+\partial_{x}(\mathbf{F}(\mathbf{W})-\tilde{\mathbf{F}}(\mathbf{W}))+\partial_{y}(\mathbf{G}(\mathbf{W})-\tilde{\mathbf{G}}(\mathbf{W}))=\mathbf{Q}(\mathbf{W}),
$$

where $\mathbf{W}$ and $\mathbf{Q}$ are the vectors of conserved variables and source terms, $\mathbf{F}$ and $\mathbf{G}$ are the convection tensor fluxes, $\tilde{\mathbf{F}}$ and $\tilde{\mathbf{G}}$ are the diffusion tensor fluxes

$$
\begin{array}{r}
\mathbf{W}=\left(\begin{array}{c}
h \\
h u \\
h v \\
h C
\end{array}\right), \quad \mathbf{Q}(\mathbf{W})=\left(\begin{array}{c}
0 \\
-g h\left(S_{0 x}-S_{f x}\right) \\
-g h\left(S_{0 y}-S_{f y}\right) \\
h Q
\end{array}\right), \\
\mathbf{F}(\mathbf{W})=\left(\begin{array}{c}
h u \\
h u^{2}+\frac{1}{2} g h^{2} \\
h u v \\
h u C
\end{array}\right), \quad \mathbf{G}(\mathbf{W})=\left(\begin{array}{c}
h v \\
h u v \\
h v^{2}+\frac{1}{2} g h^{2} \\
h v C
\end{array}\right), \\
\tilde{\mathbf{F}}(\mathbf{W})=\left(\begin{array}{c}
0 \\
0 \\
0
\end{array} \quad \tilde{\mathbf{G}}(\mathbf{W})=\left(\begin{array}{c}
0 \\
0 \\
D_{y x} \partial_{x}(h C)+D_{y y} \partial_{y}(h C)
\end{array}\right),\right.
\end{array}
$$

where $D_{x x}, D_{x y}, D_{y x}$ and $D_{y y}$ are entries of the diffusion matrix $\mathbf{D}$ assumed to be nonnegative. Remark that we have considered only a single pollutant with concentration $C$ transported by 
the shallow water flow, however the techniques presented in this paper can straightforwardly be extended to multiple pollutants. The equations (2.6) has to be solved in a bounded spatial domain $\Omega$ with smooth boundary $\Gamma$, equipped with given boundary and initial conditions. In practice, these conditions are problem dependent and their discussion is postponed for section 4 where numerical examples are discussed.

The integral form of the equations (2.6) over a fixed volume $V$ is given by

$$
\partial_{t} \int_{V} \mathbf{W} d V+\int_{V}\left(\partial_{x}(\mathbf{F}(\mathbf{W})-\tilde{\mathbf{F}}(\mathbf{W}))+\partial_{y}(\mathbf{G}(\mathbf{W})-\tilde{\mathbf{G}}(\mathbf{W}))\right) d V=\int_{V} \mathbf{Q}(\mathbf{W}) d V,
$$

that, using divergence theorem for the second integral leads to

$$
\partial_{t} \int_{V} \mathbf{W} d V+\int_{\partial V} \mathcal{F}(\mathbf{W} ; \mathbf{n}) d \sigma-\int_{\partial V} \tilde{\mathcal{F}}(\mathbf{W} ; \mathbf{n}) d \sigma=\int_{V} \mathbf{Q}(\mathbf{W}) d V,
$$

where

$$
\mathcal{F}(\mathbf{W} ; \mathbf{n})=\mathbf{F}(\mathbf{W}) n_{x}+\mathbf{G}(\mathbf{W}) n_{y}, \quad \tilde{\mathcal{F}}(\mathbf{W} ; \mathbf{n})=\tilde{\mathbf{F}}(\mathbf{W}) n_{x}+\tilde{\mathbf{G}}(\mathbf{W}) n_{y},
$$

and $\partial V$ is the surface surrounding the volume $V$. In $(2.7), \mathbf{n}=\left(n_{x}, n_{y}\right)^{T}$ denotes the unit outward normal to the surface $\partial V$.

\section{Finite Volume Non-Homogeneous Riemann Solver}

In this section we formulate the SRNH method used to solve the equations (2.7). The method uses a grid of triangular cells to facilitate grid generation and localized refinement when modeling pollutant transport in realistic waterways. A predictor-corrector stepping is used for time integration. In the predictor step, a non-conservative approach is used to determine the intermediate values over a half time step whereas in the corrector step, a fully conservative solution over a full time step is achieved by solving a series of local Riemann problems based on data from the predictor step.

\subsection{Formulation of the SRNH Scheme}

The spatial domain $\bar{\Omega}=\Omega \cup \Gamma$ is discretized by conforming triangular elements $\mathcal{T}_{i}$ as $\bar{\Omega}=\cup_{i=1}^{N e} \mathcal{T}_{i}$, with $N e$ is the total number of elements. Each triangle represents a control volume and the variables are located at the geometric centres of the cells. In the current work, a cell-centred finite volume method is formulated where all the dependent variables of the system are represented as piecewise constant in the cell as

$$
\mathbf{W}_{i}=\frac{1}{\left|\mathcal{T}_{i}\right|} \int_{\mathcal{T}_{i}} \mathbf{W} d V
$$

where $\left|\mathcal{T}_{i}\right|$ denotes the area of the element $\mathcal{T}_{i}$. Let us divide the time domain in sub-intervals $\left[t_{n}, t_{n+1}\right]$ with time stepsize $\Delta t$. Using the finite volume approach on unstructured grid (see Figure 3.1 ), the equation (2.7) is discretized as

$$
\begin{aligned}
\mathbf{W}_{i}^{n+1}= & \mathbf{W}_{i}^{n}-\frac{\Delta t}{\left|\mathcal{T}_{i}\right|} \sum_{j \in N(i)} \int_{\Gamma_{i j}} \mathcal{F}^{n+1 / 2}(\mathbf{W} ; \mathbf{n}) d \sigma+\frac{\Delta t}{\left|\mathcal{T}_{i}\right|} \sum_{j \in N(i)} \int_{\Gamma_{i j}} \tilde{\mathcal{F}}^{n+1 / 2}(\mathbf{W} ; \mathbf{n}) d \sigma- \\
& \frac{\Delta t}{\left|\mathcal{T}_{i}\right|} \int_{\mathcal{T}_{i}} \mathbf{Q}^{n+1 / 2}(\mathbf{W}) d V,
\end{aligned}
$$

where $N(i)$ is the set of neighbour cells of the cell $\mathcal{T}_{i}, \mathbf{W}_{i}^{n}$ is the average value of $\mathbf{W}$ in the cell $\mathcal{T}_{i}$ at time $t_{n}$, and $\mathbf{Q}^{n+1 / 2}$ is the averaged value of the source term in the cell $\mathcal{T}_{i}$ between the time levels $t_{n}$ 


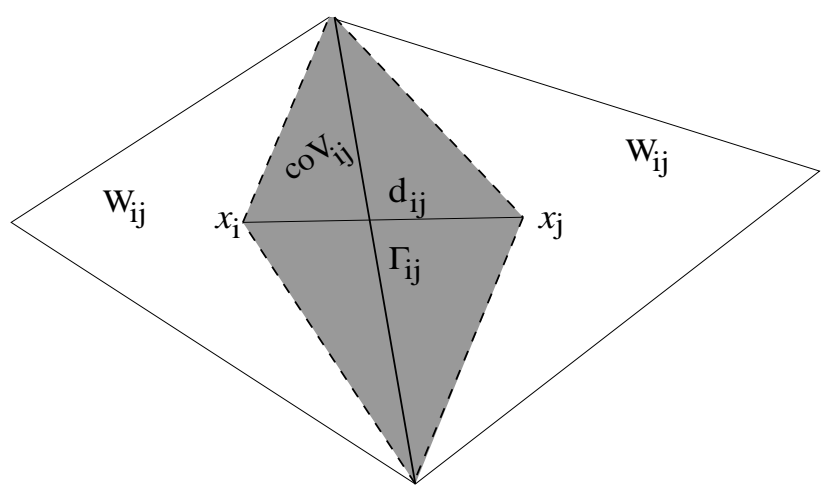

Figure 3.1: Generic triangular elements and notations.

and $t_{n+1}$. Note that the spatial discretization of (2.7) is complete when numerical reconstructions of convection and diffusion fluxes in (3.1) are selected.

Applied to the convection part in (3.1), the SRNH reconstruction yields

$$
\int_{\Gamma_{i j}} \mathcal{F}(\mathbf{W} ; \mathbf{n}) d S=\Phi\left(\mathbf{W}_{i}, \mathbf{W}_{j}, S_{i j}, \mathbf{n}_{i j}\right)\left|\Gamma_{i j}\right|,
$$

with $\left|\Gamma_{i j}\right|$ is the width of the interface $\Gamma_{i j}$ between cells $\mathcal{T}_{i}$ and $\mathcal{T}_{j}$ as shown in Figure $3.1, \mathbf{n}_{i j}$ is the outward normal to $\Gamma_{i j}, \Phi$ is the numerical flux, $\mathbf{W}_{i}$ and $\mathbf{W}_{j}$ are respectively, the left and right values of $\mathbf{W}$ at cell $\mathcal{T}_{i}$ and $\mathcal{T}_{j}$, compare Figure 3.1. Remark that the numerical SRNH flux $\Phi$ depends also on the slope $S_{i j}$ which has to be approximated. The SRNH scheme results in

$$
\begin{aligned}
\mathbf{W}_{i j}^{n} & =\frac{1}{2}\left(\mathbf{W}_{i}^{n}+\mathbf{W}_{j}^{n}\right)-\frac{\alpha_{i j}^{n}}{2 S_{i j}^{n}}\left(\mathcal{F}\left(\mathbf{W}_{j}^{n}\right)-\mathcal{F}\left(\mathbf{W}_{i}^{n}\right)\right) \cdot \mathbf{n}_{i j}+\frac{\alpha_{i j}^{n}}{2 S_{i j}^{n}} \mathbf{Q}_{i j}^{n}, \\
\mathbf{W}_{i}^{n+1} & =\mathbf{W}_{i}^{n}-\frac{\Delta t}{\left|\mathcal{T}_{i}\right|} \sum_{j \in N(i)} \Phi\left(\mathbf{W}_{i}, \mathbf{W}_{j}, S_{i j}, \mathbf{n}_{i j}\right)\left|\Gamma_{i j}\right|+\Delta t \mathbf{Q}_{i}^{n},
\end{aligned}
$$

where $\alpha_{i j}^{n}$ is a positive control parameter to be chosen according to certain stability conditions analyzed in $[32,10,9,33]$. The numerical flux is defined as

$$
\Phi\left(\mathbf{W}_{i}, \mathbf{W}_{j}, S_{i j}, \mathbf{n}_{i j}\right)=\mathcal{F}\left(\mathbf{W}_{i j}^{n}\right) \cdot \mathbf{n}_{i j},
$$

with $S_{i j}^{n}$ is the Rusanov speed

$$
S_{i j}^{n}=\max _{p}\left(\left|\lambda_{p, i}^{n}\right|,\left|\lambda_{p, j}^{n}\right|\right),
$$

where $\lambda_{p, i}^{n}$ denotes the $p$ th eigenvalue in (2.4) evaluated at the state $\mathbf{W}_{i}^{n}$. The analysis reported in $[10,32,9]$ reveals that the parameter $\alpha_{i j}^{n}$ in (3.3) can be interpreted as a diffusion coefficient in the SRNH scheme such that more numerical dissipation is added for larger values of $\alpha_{i j}^{n}$. In the present work, the diffusion coefficient is approximated in a matrix form such that a local maximum principle for the intermediate state $\mathbf{W}_{i j}^{n}$ is ensured, compare [32] for more details. Hence,

$$
\alpha_{i j}^{n}=S_{i j}^{n}\left|\nabla \mathcal{F}\left(\overline{\mathbf{W}}_{i j}^{n}\right)^{-1}\right|,
$$

where $\nabla \mathcal{F}\left(\overline{\mathbf{W}}_{i j}^{n}\right)$ is the Jacobian matrix with respect to $\mathbf{W}$, and $\overline{\mathbf{W}}_{i j}^{n}$ is approximated either by Roe's average state or simply by the mean state

$$
\overline{\mathbf{W}}_{i j}^{n}=\frac{1}{2}\left(\mathbf{W}_{i}^{n}+\mathbf{W}_{j}^{n}\right) .
$$


Using (3.4) in (3.3), the intermediate state can be reformulated as

$$
\mathbf{W}_{i j}^{n}=\frac{1}{2}\left(\mathbf{W}_{i}^{n}+\mathbf{W}_{j}^{n}\right)-\frac{1}{2} \operatorname{sgn}\left(\nabla \mathcal{F}\left(\overline{\mathbf{W}}_{i j}^{n}\right)\right)\left(\mathbf{W}_{j}^{n}-\mathbf{W}_{i}^{n}\right) \cdot \mathbf{n}_{i j}+\frac{1}{2}\left|\nabla \mathcal{F}\left(\overline{\mathbf{W}}_{i j}^{n}\right)^{-1}\right| \mathbf{Q}_{i j}^{n} .
$$

Note that an SRNH scheme with constant $\alpha_{i j}^{n}$ has been used by Benkhaldoun and Quivy [9] for hyperbolic systems with source terms. Note also that, for homogeneous systems $(\mathbf{Q}=\mathbf{0})$, the reconstruction (3.3) using (3.5) reduces to the VFRoe scheme early studied by Masella et al. [26].

It should be pointed out that the selection (3.4) leads to a first-order SRNH scheme. In order to develop second-order SRNH scheme, we use a MUSCL method incorporating slope limiters in the spatial approximation and a two step Runge-Kutta method for time integration. It is preferable to apply simple slope limiters in which the degrees of freedom $\mathbf{W}_{i}$ for a given cell $\mathcal{T}_{i}$ are compared to the average of the approximate solution over $\mathcal{T}_{i}$ and the average of the neighboring cells of the given edge. The well-established MinMod limiter can be an example of these limiter functions. The MindMod limiter is very easy to implement, but it can cause numerical smoothing of the solution. More sophisticated limiters that are less dissipative are also applicable. For instance, in the computational results presented in section 4, we have also applied the VanAlbada limiter.

To discretize the diffusion fluxes in (2.7) we adapt a Green-Gauss diamond reconstruction, see for example [12] and further references are therein. This method has been selected because it is second-order accurate, it can be applied on general unstructured adaptive grids, it does not require serious restrictions on the angles of triangles, and it can be easily incorporated in our SRNH scheme. Hence, a co-volume, $c o V_{i j}$, is first constructed by connecting the barycentres of the elements that share the edge $\Gamma_{i j}$ and its endpoints as shown in Figure 3.1. Then, in the $x$-direction, diffusion fluxes in the transport equation are evaluated at an inner edge $\Gamma_{i j}$ as

$$
\int_{\Gamma_{i j}} D \partial_{x}(h C) n_{x} d \sigma=\frac{D}{\left|c o V_{i j}\right|} \sum_{\epsilon \in \partial c o V_{i j}} \frac{(h C)_{N_{1}}-(h C)_{N_{2}}}{2} \int_{\epsilon} n_{x} \epsilon d \sigma,
$$

where $N_{1}$ and $N_{2}$ are the nodes of the edge $\epsilon$ on the surface $\partial c o V_{i j},(h C)_{N_{1}}$ and $(h C)_{N_{2}}$ are the values of the state $h C$ in the node $N_{1}$ and $N_{2}$, respectively. The discretization in $y$-direction of diffusion fluxes is carried out in an analogous manner. Note that, in (3.7) we have assumed constant diffusion coefficient. In the case of space dependent diffusion, the coefficient $D$ in (3.7) should be replaced by

$$
\frac{D_{N_{1}}+D_{N_{2}}+D_{N_{3}}+D_{N_{4}}}{4}
$$

with $D_{N_{k}}, k=1, \ldots, 4$, are values of the diffusion coefficient $D$ at the co-volume nodes $N_{k}$ approximated by linear interpolation from the values on the cells sharing the same vertex $N_{k}$.

\subsection{Features of the SRNH Scheme}

\subsubsection{Discretization of the source terms}

In the predictor step of the SRNH reconstruction (3.3), the source terms are treated using the ideas developed in $[1,28]$. First, we project the shallow water equations on the local cell outward normal $\eta$ and tangential $\tau=\eta^{\perp}$ as follows

$$
\begin{aligned}
\partial_{t} h+\partial_{\eta}\left(h u_{\eta}\right) & =0 \\
\partial_{t}\left(h u_{\eta}\right)+\partial_{\eta}\left(h u_{\eta}^{2}+\frac{1}{2} g h^{2}\right) & =-g h \partial_{\eta} Z, \\
\partial_{t}\left(h u_{\tau}\right)+\partial_{\eta}\left(h u_{\eta} u_{\tau}\right) & =0 \\
\partial_{t}(h C)+\partial_{\eta}\left(h C u_{\eta}\right) & =0,
\end{aligned}
$$


where $u_{\eta}=(u, v) \cdot \eta$ and $u_{\tau}=(u, v) \cdot \tau$ are the normal and tangential velocity, respectively. In this case, the predictor step becomes

$$
\mathbf{U}_{i j}^{n}=\frac{1}{2}\left(\mathbf{U}_{i}^{n}+\mathbf{U}_{j}^{n}\right)-\frac{1}{2} \operatorname{sgn}\left(\nabla \mathbf{F}_{\eta}(\overline{\mathbf{U}})\right)\left(\mathbf{U}_{j}^{n}-\mathbf{U}_{i}^{n}\right)+\frac{1}{2}\left|\nabla \mathbf{F}_{\eta}(\overline{\mathbf{U}})^{-1}\right| \mathbf{Q}_{i j}^{n},
$$

with $\operatorname{sgn}(\mathbf{A})$ stands for the sign matrix of $\mathbf{A}$ and

$$
\mathbf{U}=\left(\begin{array}{c}
h \\
h u_{\eta} \\
h u_{\tau} \\
h C
\end{array}\right), \quad \mathbf{F}_{\eta}(\mathbf{U})=\left(\begin{array}{c}
h u_{\eta} \\
h u_{\eta}^{2}+\frac{1}{2} g h^{2} \\
h u_{\eta} u_{\tau} \\
h C u_{\eta}
\end{array}\right), \quad \mathbf{Q}_{i j}^{n}=-g \frac{h_{i}+h_{j}}{2}\left(Z_{j}-Z_{i}\right)\left(\begin{array}{c}
0 \\
1 \\
0 \\
0
\end{array}\right)
$$

In (3.9), $\overline{\mathbf{U}}$ is the Roe average state given by

$$
\overline{\mathbf{U}}=\frac{1}{2}\left(h_{i}+h_{j}\right)\left(\begin{array}{c}
1 \\
\frac{u_{i} \sqrt{h_{i}}+u_{j} \sqrt{h_{j}}}{\sqrt{h_{i}}+\sqrt{h_{j}}} \eta_{x}+\frac{v_{i} \sqrt{h_{i}}+v_{j} \sqrt{h_{j}}}{\sqrt{h_{i}}+\sqrt{h_{j}}} \eta_{y} \\
-\frac{u_{i} \sqrt{h_{i}}+u_{j} \sqrt{h_{j}}}{\sqrt{h_{i}}+\sqrt{h_{j}}} \eta_{y}+\frac{v_{i} \sqrt{h_{i}}+v_{j} \sqrt{h_{j}}}{\sqrt{h_{i}}+\sqrt{h_{j}}} \eta_{x} \\
\frac{C_{i} \sqrt{h_{i}}+C_{j} \sqrt{h_{j}}}{\sqrt{h_{i}}+\sqrt{h_{j}}}
\end{array}\right) .
$$

The sign and inverse matrices of the Jacobian are defined as

$$
\operatorname{sgn}\left(\nabla \mathbf{F}_{\eta}(\overline{\mathbf{U}})\right)=\mathcal{R}(\overline{\mathbf{U}}) \operatorname{sgn}(\Lambda(\overline{\mathbf{U}})) \mathcal{R}^{-1}(\overline{\mathbf{U}}), \quad\left|\nabla \mathbf{F}_{\eta}(\overline{\mathbf{U}})^{-1}\right|=\mathcal{R}(\overline{\mathbf{U}})\left|(\Lambda(\overline{\mathbf{U}}))^{-1}\right| \mathcal{R}^{-1}(\overline{\mathbf{U}}),
$$

where $\mathcal{R}(\overline{\mathbf{U}})$ is the left eigenvector matrix reconstructed from the eigenvalues

$$
\lambda_{1}=u_{\eta}, \quad \lambda_{2}=u_{\eta}+\sqrt{g h}, \quad \lambda_{3}=u_{\eta}-\sqrt{g h}, \quad \lambda_{4}=u_{\eta} .
$$

Once the state $U_{i j}^{n}$ is computed, the state $\mathbf{W}_{i j}^{n}$ is recovered by using the transformations $v=$ $\left(u_{\tau}, u_{\eta}\right) \cdot \eta$ and $u=\left(u_{\tau}, u_{\eta}\right) \cdot \tau$. The source term approximation $\mathbf{Q}_{i}^{n}$ in the corrector step is reconstructed in such a way to satisfy the C-property, see [10,32]. This construction provides the exact approximations of flow variables in the computational domain for the stationary problems.

\subsubsection{Adaptivity procedure}

The mesh generation is based on the Delaunay triangulation [18], which uses a curvature-dependent generation strategy designed to produce smaller elements in regions of high curvature in the spatial domain. In order to improve the efficiency of the SRNH scheme, we have performed a mesh adaptation to construct an optimal mesh able to capture the small hydraulic and pollutant features without relying on extremely fine grid in smooth regions far from concentration or hydraulic jumps. In the present work, this goal is achieved by using an error indicator for the concentration of pollutant. This residual only requires information from solution values within a single element at a time and it is easily calculated. Other adaptation techniques based on the estimation of gradients such as those studied by Babuska et al. [8] can also be applied. However, these error estimations can be computationally very demanding since a global solution step is needed to project the gradients on a linear basis.

This error indicator is numerically estimated using residual values with integration points at each node of the element. The error indicator is then incorporated into an adaptive SRNH algorithm employing triangular elements. The adaption is based on the element refinement indicator tolerance 


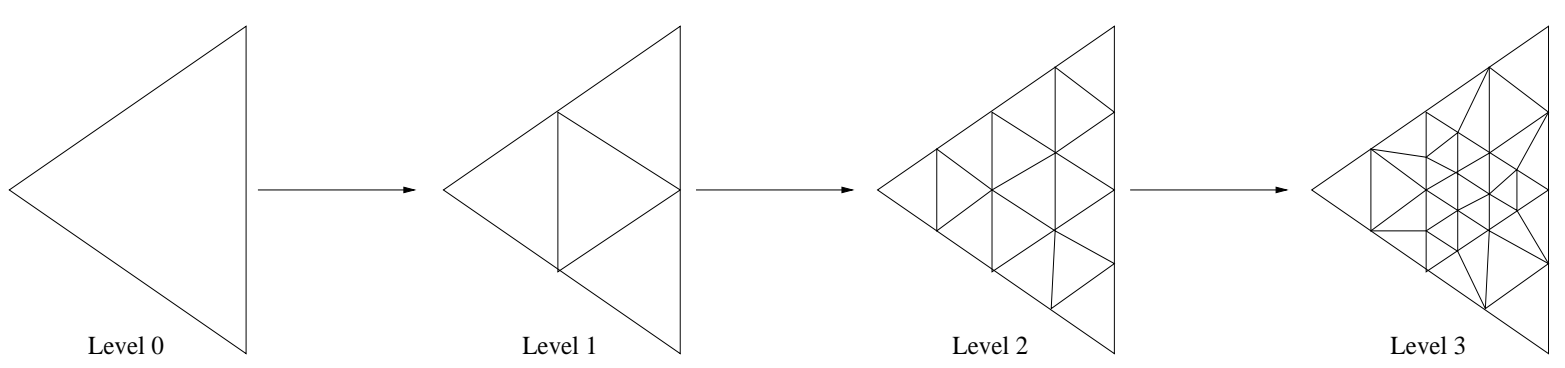

Figure 3.2: Example of a three-level refining for triangular elements.

set by the user. In our implementation, the mesh is adapted using local mesh modification operators that enable fast and accurate solution transfers. In addition, a coarsening step consists of merging four cells to obtain a child cell. Thus, starting from a mesh level $l$, made of $N^{(l)}$ cells, the next mesh level contains $N^{(l+1)}=N^{(l)} / 4$ cells. Clearly, this process can be repeated as long as $N^{(l)}$ is an even number. Whenever one of the number of cells is odd, the coarsening automatically switches to a one-dimensional coarsening procedure in which only two cells are merged to make a child cell. It is clear that the procedure is optimal when $N^{(l)}$ is power of 2, compare Figure 3.2 for an illustration. Similar algorithms have been developed by Elmahi et al. [12] for adaptive finite volume solution of a combustion system.

\subsubsection{Boundary conditions}

The treatment of boundary conditions in the SRNH scheme is performed using similar techniques described in [29]. For the computational examples considered in this paper, boundary conditions are enforced on the corrector solution by computing fluxes at boundaries. On the predictor solution and the slopes of dependent variables, boundary conditions are enforced in boundary cells by setting to the corresponding values of the adjacent inner cells. When slopes are based on vertex values, the solution at boundary vertices is computed by interpolation from two neighboring centroids. When slopes are based on centroid values, the three points used to estimate the slopes are the centroid and two neighboring centroids inside the model domain. For further details on the implementation of boundary conditions for the SRNH scheme we refer to $[29,32]$.

\section{Numerical Results and Applications}

Three test examples are selected to check the accuracy and performance of the proposed SRNH scheme. The first example is used to assess different features of our scheme such as accuracy in smooth regions and discontinuous resolution in a dam-break problem on nonflat topography. The second example solves a pollutant transport in a squared cavity with smooth bottom. As a third example we apply our adaptive SRNH scheme for a pollutant transport in the strait of Gibraltar. All the results presented in this section are computed with variable time stepsizes $\Delta t$ adjusted at each step according to

$$
\Delta t=C r \cdot \min \left(\frac{\Delta x_{i}}{\left|u_{i}\right|+\sqrt{g h_{i}}}, \frac{\Delta y_{i}}{\left|v_{i}\right|+\sqrt{g h_{i}}}\right)
$$

where $\Delta x_{i}$ and $\Delta y_{i}$ are respectively, the dimensions of cell $i$ in the $x$ - and $y$-direction, and $C r$ is the Courant number set to 0.8 for all test cases to ensure stability. Three-level refining is used and the gravitational acceleration is fixed to $g=9.81 \mathrm{~m} / \mathrm{s}^{2}$ for all the examples presented here. 


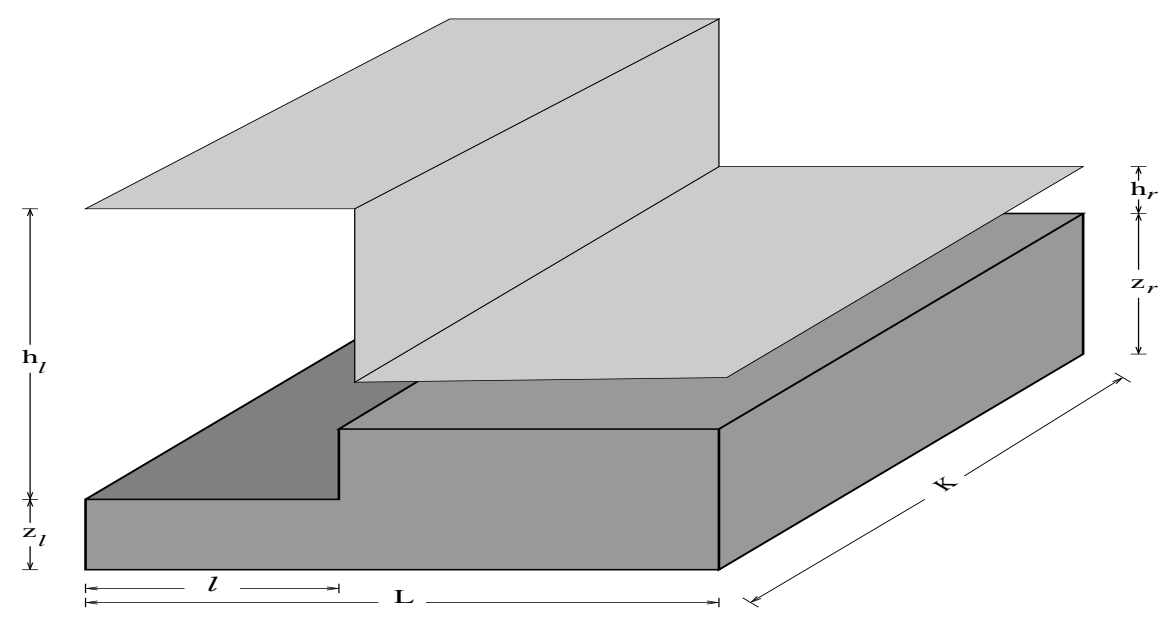

Figure 4.1: Geometrical description of shallow water flow past a forward facing step.

\subsection{Shallow Water Flow over a Forward Facing Step}

We consider a two-dimensional dam-break problem over a forward facing step. The computational domain is a rectangular channel with dimensions $L \times K$ and the length before the step is $l$ as depicted in Figure 4.1. The bottom bed is assumed to be frictionless $\left(S_{f x}=S_{f y}=0\right)$ and the bed elevation is specified as

$$
Z(x, y)=\left\{\begin{array}{lll}
z_{l}, & \text { if } & x \leq l \\
z_{r}, & \text { if } & x>l
\end{array}\right.
$$

Initial conditions are given by

$$
h(0, x, y)=\left\{\begin{array}{ll}
h_{l}, & \text { if } x \leq l, \\
h_{r}, & \text { if } x>l,
\end{array} \quad u(0, x, y)=\left\{\begin{array}{ll}
u_{l}, & \text { if } x \leq l, \\
u_{r}, & \text { if } \quad x>l,
\end{array} \quad v(0, x, y)=0 .\right.\right.
$$

This test example is very interesting since it includes most of flow structures such as shocks, rarefaction waves and contact discontinuities. Recall that in the framework of shallow water equations applied to dam-break problems, an important dimensionless parameter is the Froude number $\mathrm{Fr}$, defined as the ratio between the water velocity and the celerity $c=\sqrt{g h}$. The Froude number characterizes the subcritical $(F r<1)$ or supercritical $(F r>1)$ regime of the flow. Both flow regimes are examined for this test example.

First we run the SRNH scheme for the subcritical case. This test aims to compare the performance of the SRNH scheme to the Vázquez scheme [36] widely known in the literature. We used $L=12 \mathrm{~m}, K=1 \mathrm{~m}, l=6 \mathrm{~m}, z_{l}=0 \mathrm{~m}, z_{r}=1 \mathrm{~m}, h_{l}=5 \mathrm{~m}, h_{r}=2 \mathrm{~m}$, and $u_{l}=u_{r}=0 \mathrm{~m} / \mathrm{s}$. An unstructured grid with 14471 elements and 7657 nodes is used for both schemes. The water free surface and the water discharge are illustrated in Figure 4.2 at time $t=0.5 \mathrm{~s}$. In Figure 4.3 we show cross sections at the channel mid-length $(y=K / 2)$ for the obtained results. We present the water head $h+Z+\frac{u^{2}}{2 g}$, the water free surface $h+Z$, the water discharge $h u$, and the Froude number $\mathrm{Fr}$.

As can be seen in Figure 4.3, both schemes exhibit a slight peak between the dam and the shock just on region where the bottom step is localized, compare the zooming plots in Figure 4.3 for the water head and the water discharge. However, the amplitude of the peak is most pronounced in the Vázquez scheme. In this test case, the original Vázquez scheme shows a high level of oscillations due to the irregular bottom which can not be handled because the imbalance between the source and flux terms, while a considerable improvement is observed with our SRNH scheme. 

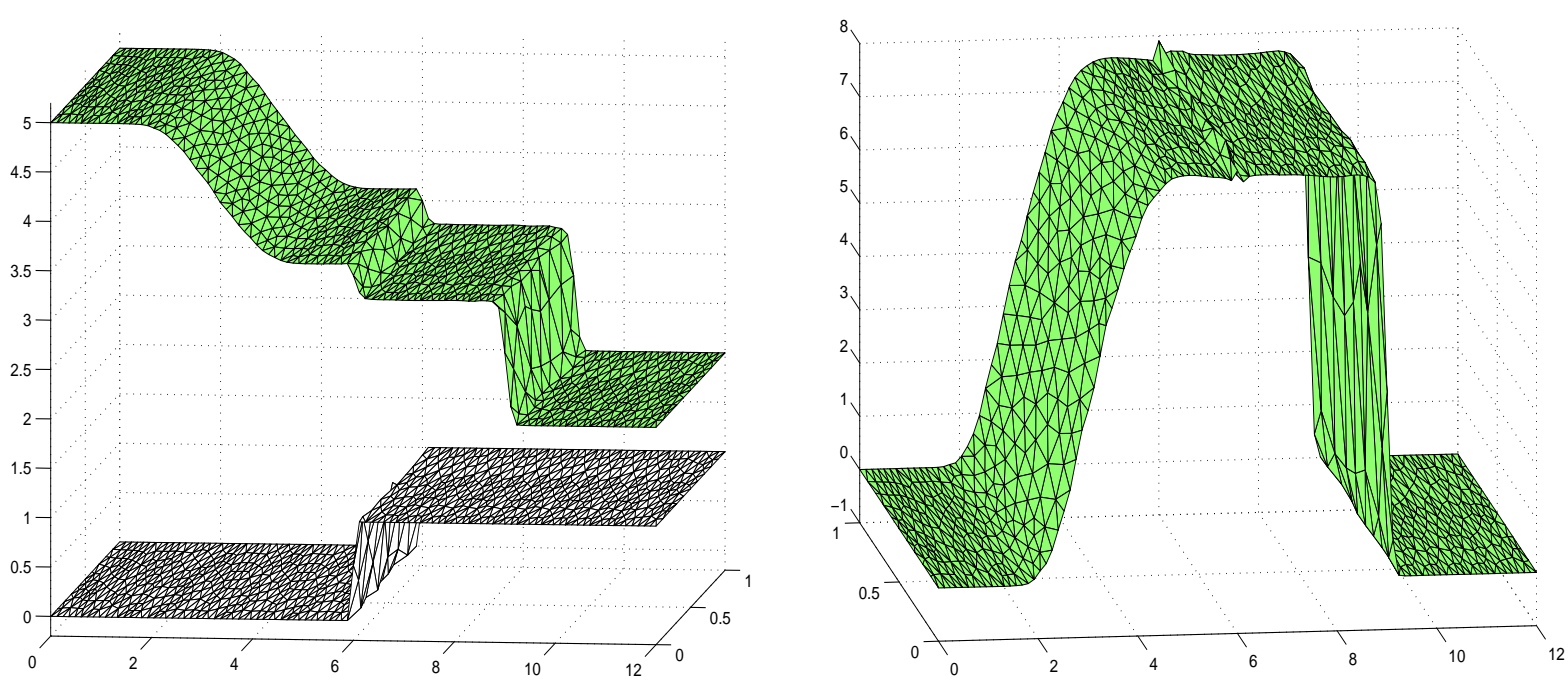

Figure 4.2: Water free surface and water discharge for the subcritical test case at $t=0.5 \mathrm{~s}$.
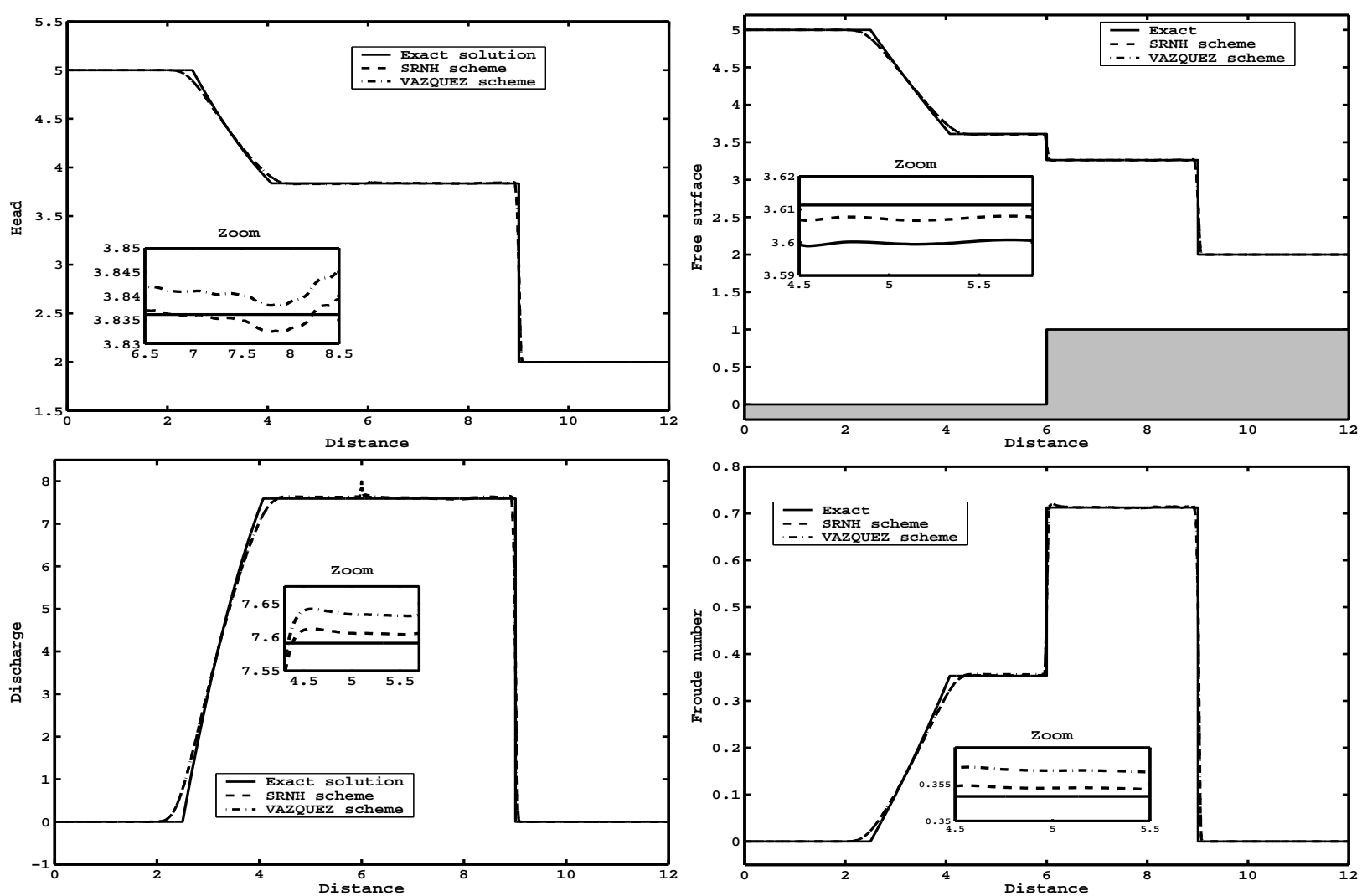

Figure 4.3: Subcritical flow past a forward facing step at time $t=0.5 \mathrm{~s}$. 

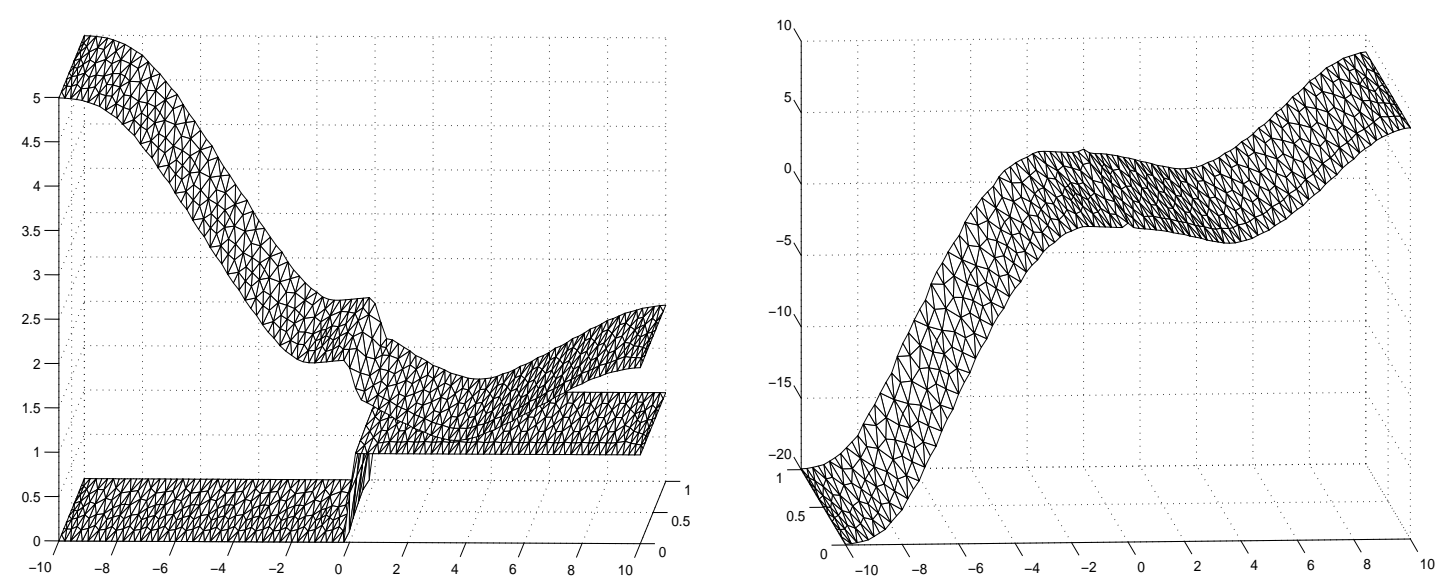

Figure 4.4: Water free surface and water discharge for the transcritical test case at $t=0.7 \mathrm{~s}$.

In the second run, we examine the accuracy between the first-order SRNH and the second-order SRNH schemes for the transcritical case. The following set of parameters is chosen $L=20 \mathrm{~m}$, $K=1 \mathrm{~m}, l=10 \mathrm{~m}, z_{l}=0 \mathrm{~m}, z_{r}=1 \mathrm{~m}, h_{l}=5 \mathrm{~m}, h_{r}=1 \mathrm{~m}, u_{l}=-4 \mathrm{~m} / \mathrm{s}$, and $u_{r}=9 \mathrm{~m} / \mathrm{s}$. The MinMod limiter is used and results are presented at time $t=0.7 \mathrm{~s}$ using an unstructured fixed grid with 17152 elements and 8793 nodes. The obtained results are displayed in Figure 4.4 and Figure 4.5. As in the previous run we present cross sections at the channel mid-length $(y=K / 2)$ for the water free surface, water head, water discharge and Froude number. It is known that roll waves or discontinuous periodic traveling waves occur in open channel flow problems when the Froude number becomes larger than two. For such flow conditions, the water flow can break to series of waves or bores that are separated by smooth flow in staircase pattern. In our results these features are clearly observed and accurately resolved by the SRNH scheme.

As can be seen in Figure 4.5, the water free surface is poorly simulated by the first-order SRNH scheme. This is not the case for the second-order SRNH scheme, which produces accurate results. As expected, the first-order SRNH scheme introduces a stronger numerical diffusion and large phase error than the second-order SRNH scheme. In summary, the SRNH scheme performs well for this dam-break problem in subcritical and transcritical flow regimes.

\subsection{Pollutant Transport in a Squared Cavity}

Our next example is a pure advection $(\mathbf{D} \equiv \mathbf{0})$ of a pollutant transport in a squared cavity with smooth topography as described in [21, 2]. The flow domain is a $9000 m \times 9000 m$ squared channel with bottom slopes

$$
S_{0 x}=S_{0 y}=-0.001 .
$$

The Manning resistance coefficient is set to $\eta=0.025 \mathrm{~s} / \mathrm{m}^{1 / 3}$. As in [21], we impose uniform flow velocities $u=v=0.5 \mathrm{~m} / \mathrm{s}$ and the uniform flow water depth as initial condition. The initial condition for the pollutant concentration is given by the superposition of two Gaussian pulses centered respectively, in $\left(x_{1}=1400 \mathrm{~m}, y_{1}=1400 \mathrm{~m}\right)$ and $\left(x_{2}=2400 \mathrm{~m}, y_{2}=2400 \mathrm{~m}\right)$,

$$
C(0, x, y)=C_{1} \exp \left(-\frac{\left(x-x_{1}\right)^{2}+\left(y-y_{1}\right)^{2}}{\sigma_{1}^{2}}\right)+C_{2} \exp \left(-\frac{\left(x-x_{2}\right)^{2}+\left(y-y_{2}\right)^{2}}{\sigma_{2}^{2}}\right),
$$

where $C_{1}=10, C_{2}=6.5$ and $\sigma_{1}=\sigma_{2}=264$. As boundary conditions we use transparent flow conditions at all cavity boundaries. It is easy to check that the pollutant concentration is a wave 

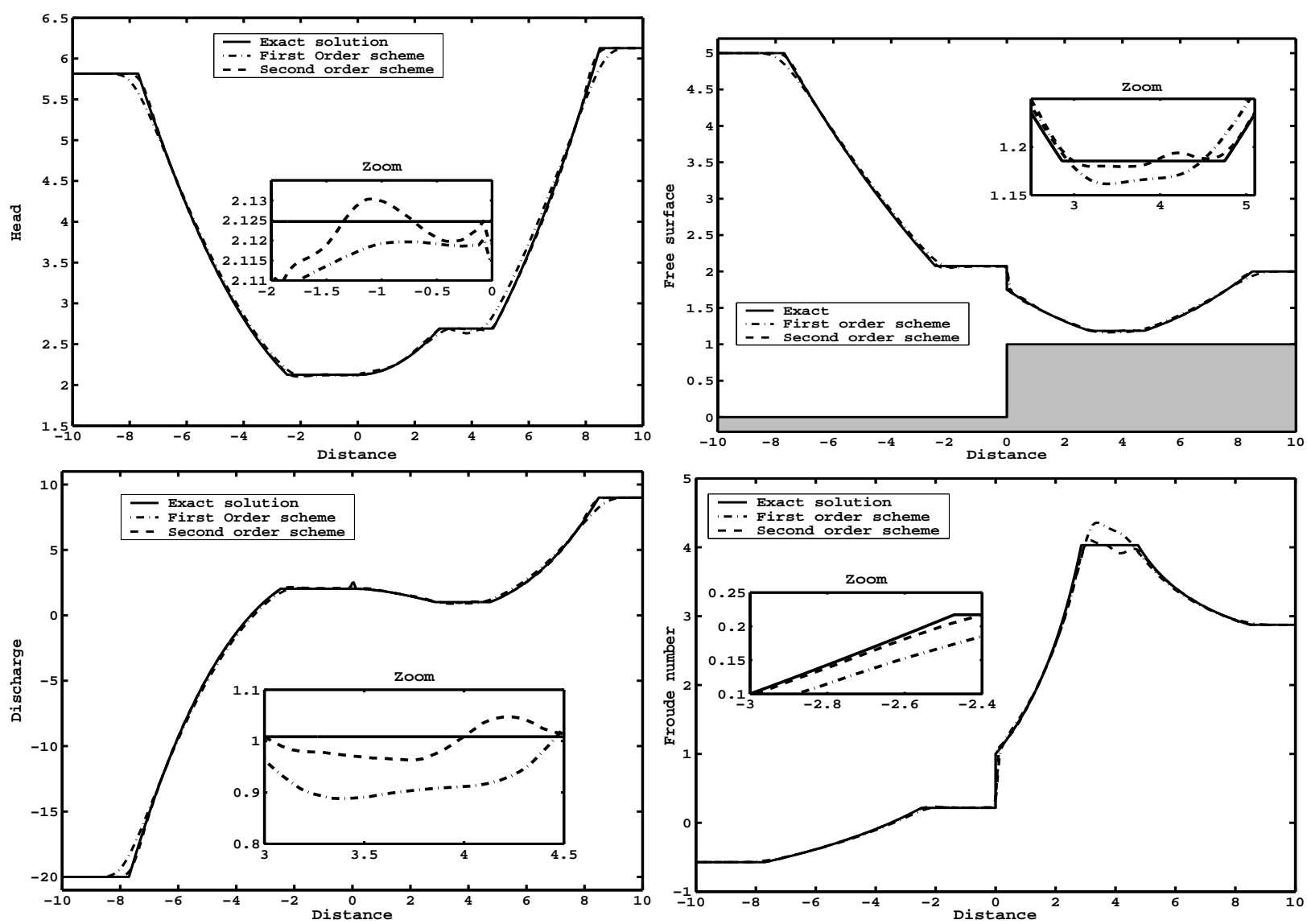

Figure 4.5: Transcritical flow past a forward facing step at time $t=0.7 \mathrm{~s}$. 
that moves along the diagonal cross-section $x=y$ preserving its shape with the constant speed $u=v=0.5 \mathrm{~m} / \mathrm{s}$.

This problem has an interesting structure and will be used to verify our adaptive SRNH scheme namely, (i) verify if the adaptation methodology is able to compute the right speed of the concentration waves, and (ii) verify that adaptive refinement is computationally cheaper than fixed mesh for a given level of solution resolution. Figure 4.6 shows the adapted meshes and plots of the pollutant concentration $C$ at times $t=1628,5235$ and $9600 \mathrm{~s}$. A simple inspection of these results shows that the pollutant shape is accurately preserved along its advection direction. Another important result is that positions of the concentration waves are not deteriorated by the multiple mesh adaptations. Our adaptive SRNH scheme accurately approximates the solution to this pollutant transport problem. In addition, the comparison with similar numerical results available in the literature $[21,2]$ on the same test case is also satisfactory. It should be stressed that, due to grid adaptation the final mesh at $t=9600 \mathrm{~s}$ consists of 14716 cells only compared to a SRNH scheme on fixed mesh. This results in a significant reduction of computational costs, see Table 4.1.

In order to get a closer comparison between the computed concentration by SRNH schemes and the analytical solution, we present in Figure 4.7 cross sections of the pollutant concentration at the main diagonal $(x=y)$ for the considered simulation times. In this figure, we have included results obtained by the SRNH scheme using MinMod and VanAlbada as limiter functions. It is evident that solutions computed using the SRNH scheme with MinMod limiter seem to be deteriorated by excessive numerical diffusion. This effect is more visible as the simulation time increases. In contrast, the SRNH scheme with VanAlbada limiter tends to move numerical diffusion of the solutions at the local extrema. There is a good agreement between the analytical solutions and those obtained using SRNH scheme with VanAlbada limiter. No local undershoots or overshoots have been detected in the pollutant concentration in presence of steep gradients during the simulation process.

Our next concern is to ascertain the behavior of adaptation procedure in the SRNH scheme. To this end we summarize in Table 4.1 some comparative results obtained for this test example at time $t=9600 \mathrm{~s}$ using fixed and adaptive meshes. All tests have the same effective discretization with respect to the smallest grid size in computational meshes. In this table we list the mesh statistics, minimum and maximum values of the pollutant concentration, residuals in the pollutant concentration, and the CPU times on a Pentium $800 \mathrm{MHz}$ with $256 \mathrm{MB}$ RAM personal computer. The clear indication from Table 4.1 is that the SRNH scheme with VanAlbada limiter overcomes the SRNH scheme with MinMod limiter on both, fixed and adaptive meshes. It is also clear that the residual in the pollutant concentration noticeably decays for the SRNH scheme with VanAlbada limiter resulting in a significant reduction of cells in associated meshes.

An examination of the CPU times in Table 4.1 reveals that, SRNH scheme on fixed meshes requires less computational work than its adaptive counterpart. For all the results presented, the computational effort needed for the SRNH scheme with MinMod limiter is almost twice the one needed for the SRNH scheme with VanAlbada limiter. This is attributed to the high accuracy achieved in results obtained by SRNH scheme with VanAlbada limiter which affects the number of cells in the adaptive procedure. In terms of minimum and maximum values of the pollutant concentration, the results obtained by SRNH scheme with VanAlbada limiter are more accurately computed than those obtained by other schemes. In all our computations, we have found that the SRNH scheme on fixed meshes with MinMod and VanAlbada limiter lost respectively, about $78 \%$ and $32 \%$ of the initial mass, whereas the adaptive SRNH scheme with VanAlbada limiter was roughly mass conservative (it lost less than $2 \%$ ). Apparently, the overall pollutant transport is conserved with no nonphysical oscillations appearing in the results by SRNH scheme with VanAlbada limiter that would alter the pollutant concentration. 

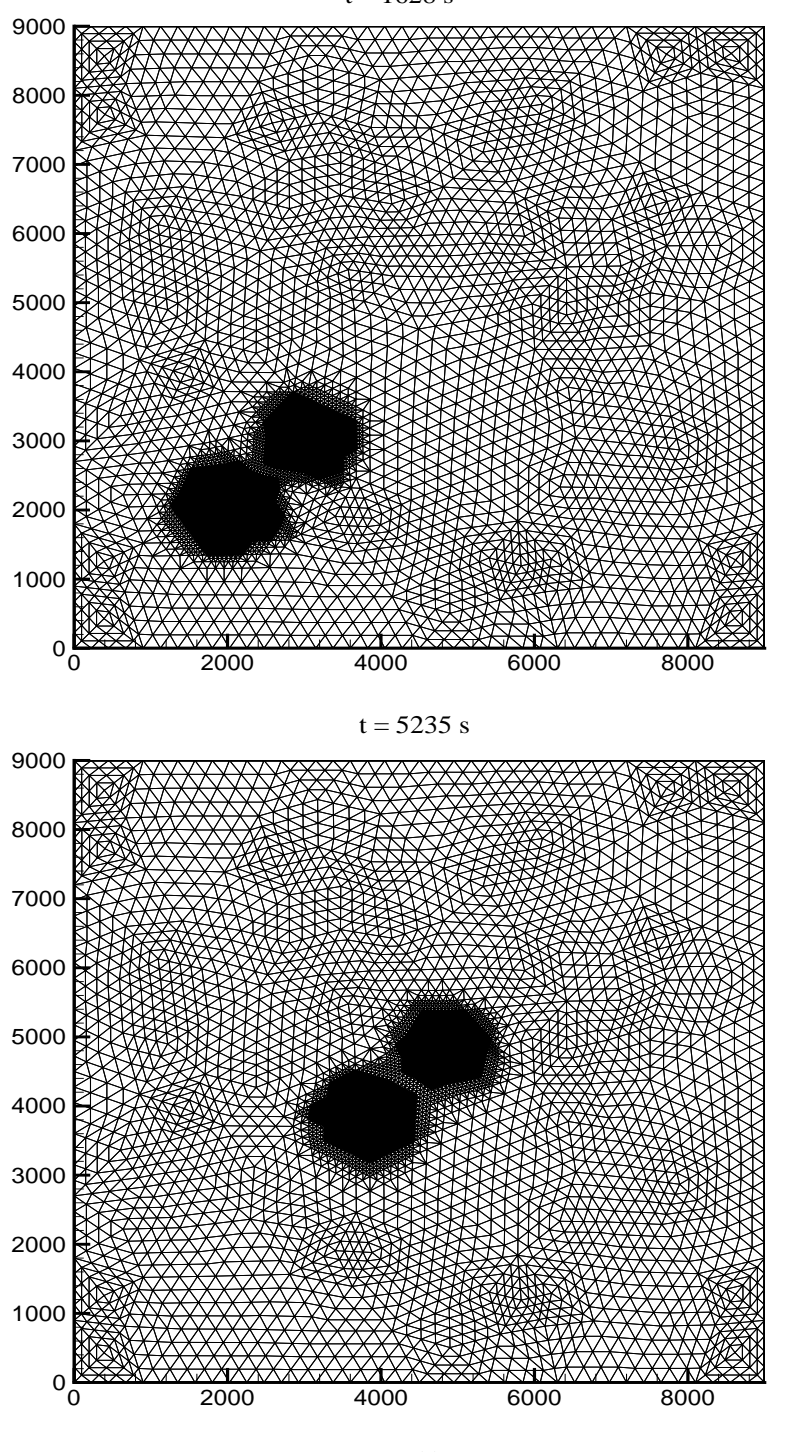

$t=9600 s$

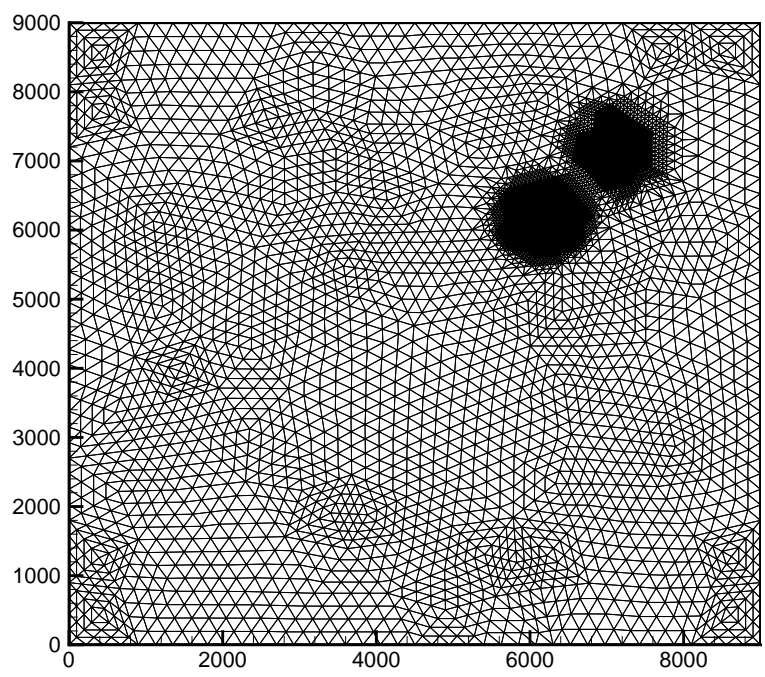

$t=1628 \mathrm{~s}$
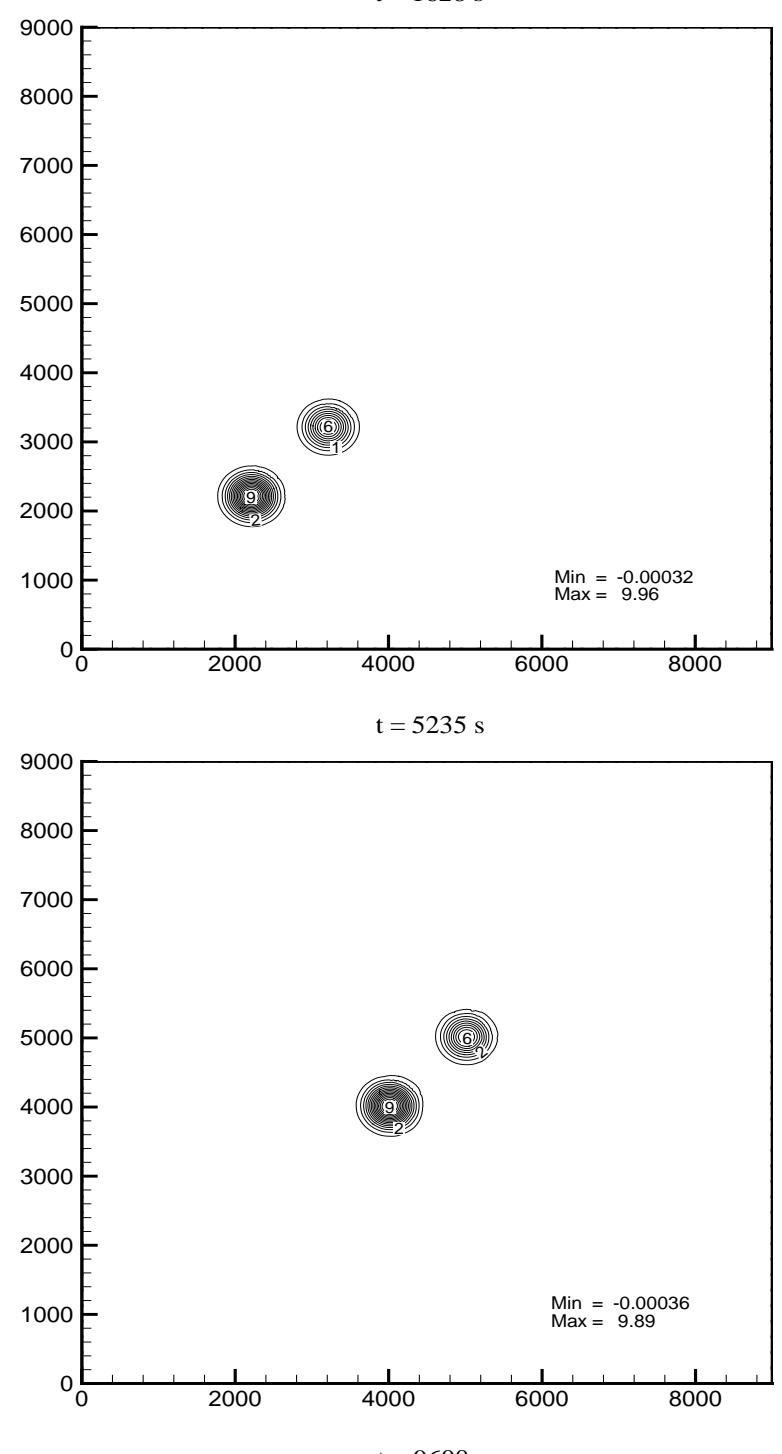

$\mathrm{t}=9600 \mathrm{~s}$

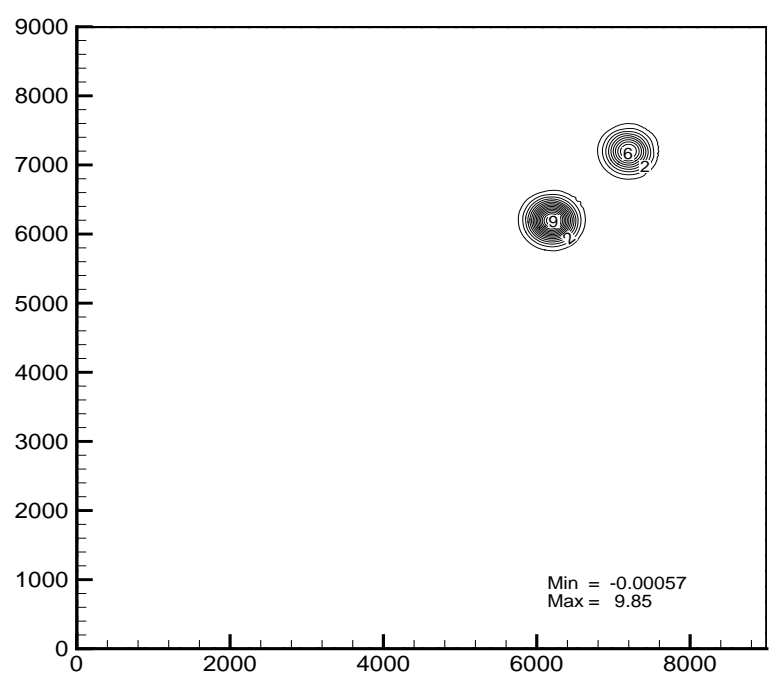

Figure 4.6: Adapted meshes (left) and contours of pollutant concentration (right) at three simulation times: From top to bottom $t=1628 s, 5235 s$ and $9600 s$. 

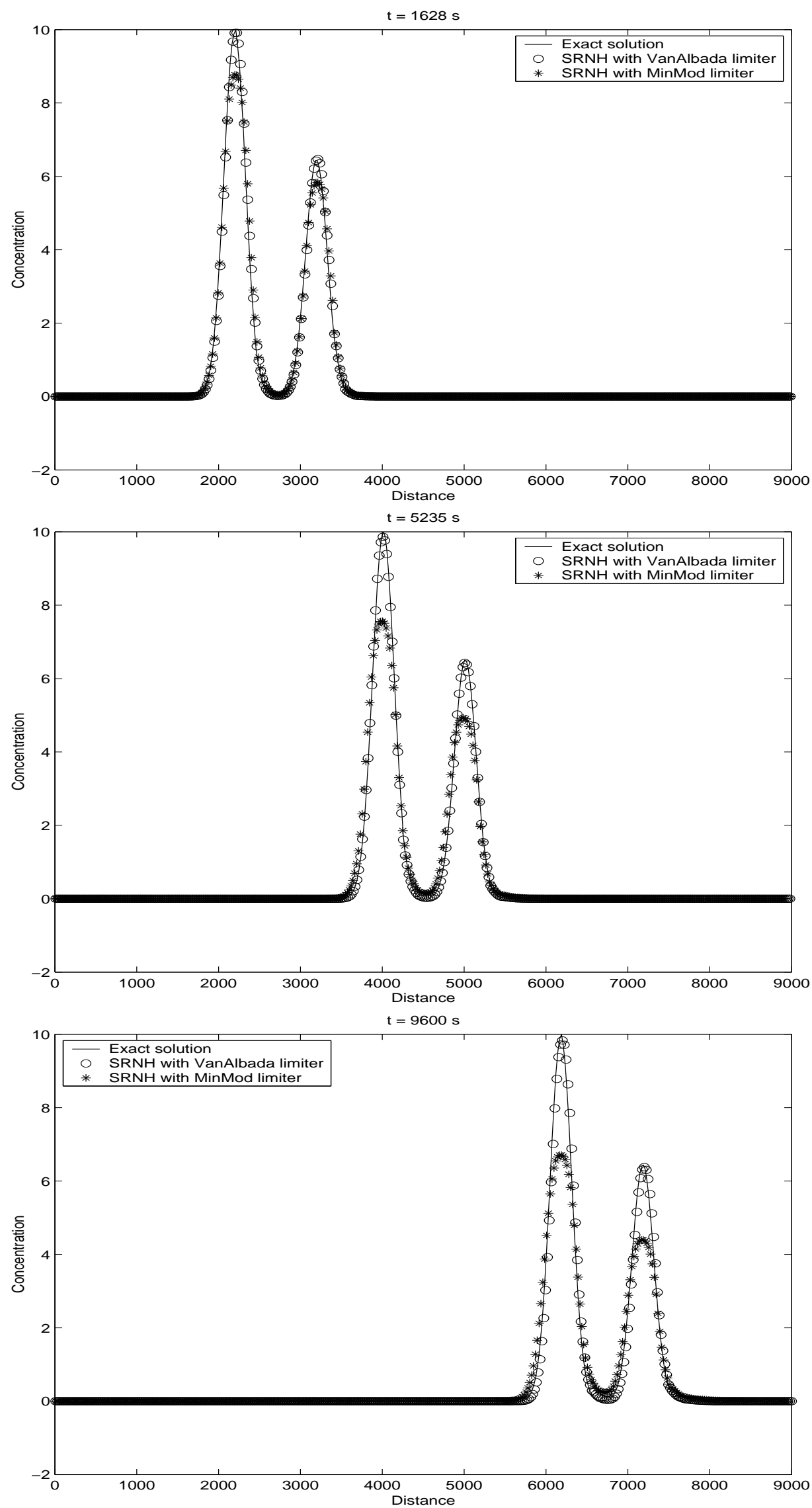

Figure 4.7: Cross sections of the pollutant concentration at the main diagonal $(x=y)$. 
Table 4.1: Performance of SRNH schemes using fixed and adaptive meshes for the pollutant transport in a squared cavity at $t=9600 \mathrm{~s}$. The CPU times are given in seconds.

\begin{tabular}{lccccccc} 
& & \multicolumn{2}{c}{ SRNH with MinMod limiter } & & \multicolumn{2}{c}{ SRNH with VanAlbada limiter } \\
\cline { 7 - 8 } \cline { 6 - 7 } & Exact & Fixed mesh & Adaptive mesh & & Fixed mesh & Adaptive mesh \\
\hline \# of elements & - & 21376 & 16154 & & 21376 & 14716 \\
\# of nodes & - & 10849 & 8158 & & 10849 & 7439 \\
Minimum of $C$ & 0.0 & 0.0 & 0.0 & & -0.2 & -0.00057 \\
Maximum of $C$ & 10.0 & 2.18 & 6.72 & & 6.8 & 9.85 \\
Residual in $C$ & - & 1.387 & 0.244 & & 0.309 & 0.026 \\
CPU time & - & 2457 & 4837 & & 1771 & 3223 \\
\hline
\end{tabular}
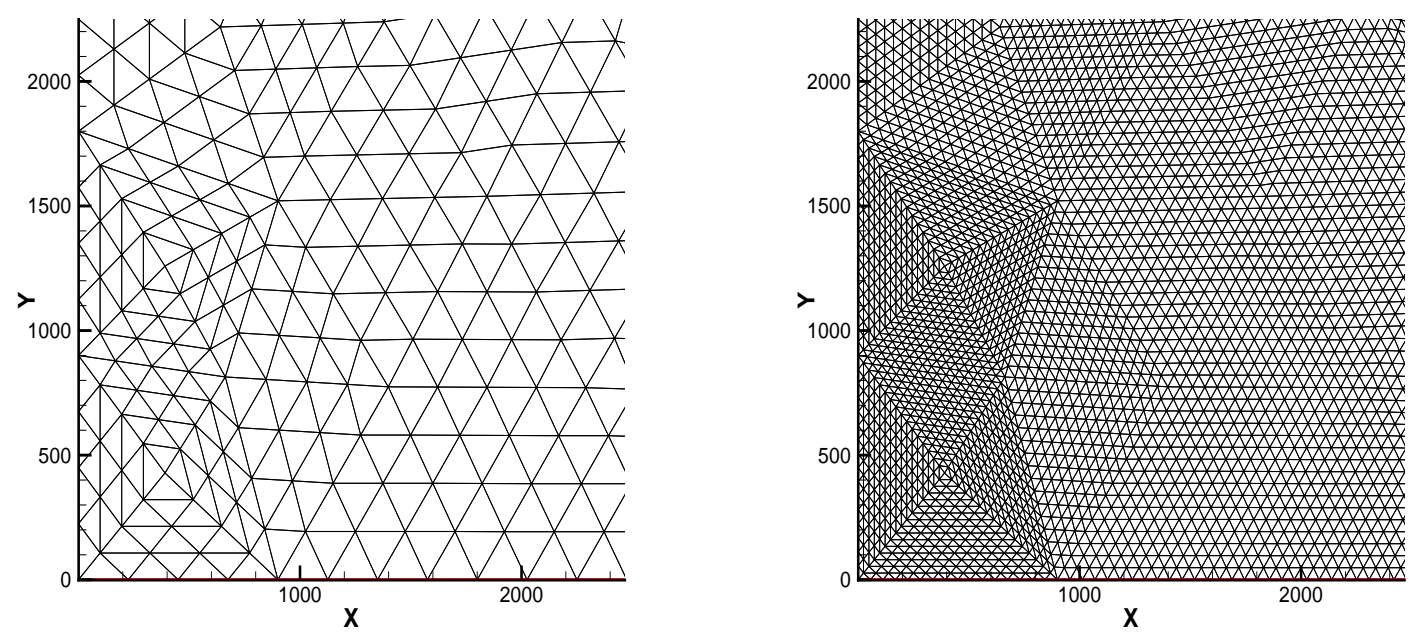

Figure 4.8: Coarse and fine fixed meshes. Mesh A (left) and Mesh B (right). 
Table 4.2: Performance of SRNH schemes with VanAlbada limiter using fixed uniform meshes for the pollutant transport in a squared cavity at $t=9600 \mathrm{~s}$. The CPU times are given in seconds.

\begin{tabular}{lccc} 
& Exact & Mesh A & Mesh B \\
\hline \# of elements & - & 5344 & 85504 \\
\# of nodes & - & 2753 & 43073 \\
Minimum of $C$ & 0.0 & -0.28 & -0.0054 \\
Maximum of $C$ & 10.0 & 3.59 & 9.12 \\
Residual in $C$ & - & 0.814 & 0.083 \\
CPU time & - & 207 & 12144 \\
\hline
\end{tabular}

As a final remark, we want to comment on the performance of the SRNH scheme with VanAlbada limiter on fixed uniform meshes. Here, we consider a coarse mesh (Mesh A) with uniform grid spacing $\Delta x=\Delta y=225 \mathrm{~m}$, and a fine mesh (Mesh B) with $\Delta x=\Delta y=56.25 \mathrm{~m}$, see Figure 4.8. In Figure 4.9 we present the cross sections of the pollutant concentration at times $t=1628$, 5235 and $9600 \mathrm{~s}$. The residual, maximum and minimum values in the pollutant concentration are listed in Table 4.2. For computations on Mesh A, we observe undershoots in the vicinity of steep gradients of concentration. These undershoots are moved from the results obtained on Mesh B, but these results are still far from the analytical solutions. It is clear that SRNH scheme on the considered fixed uniform meshes yields much larger numerical diffusion than the SRNH scheme on adaptive meshes. In fact, the residuals in the pollutant concentration obtained using the SRNH scheme on Mesh A and Mesh B are respectively, 31 and 3 times larger than the residual obtained by adaptive SRNH scheme with VanAlbada limiter. By analyzing the cross-section plots in Figure 4.9 and Figure 4.7 and the results presented in Table 4.2 and Table 4.1 we confirm that the adaptive SRNH scheme with VanAlbada limiter performs very well for this pollutant transport problem.

Taking all factors into account, we conclude that the adaptive SRNH scheme with VanAlbada limiter demonstrates higher monotone and non-oscillatory properties than other SRNH schemes. More importantly, a balance between efficiency and accuracy in SRNH schemes benefits the adaptive SRNH scheme with VanAlbada limiter, since the additional cost required for the adaptation procedure is minimal while the results obtained by adaptive SRNH schemes are more accurate than those obtained by SRNH schemes on fixed meshes.

\subsection{Pollutant Transport in the Strait of Gibraltar}

The strait of Gibraltar (see left plot in Figure 4.10) is bounded to the north and south by the Iberian and African continental forelands, and to the west and east by the Atlantic Ocean and the Mediterranean sea, respectively. The basic circulation in the strait of Gibraltar consists of an upper layer of cold fresh surface Atlantic water and an opposite deep current of warmer salty Mediterranean outflowing water, compare $[23,6,16]$. The system is about $60 \mathrm{Km}$ long between its west Barbate-Tangier section and its east Gibraltar-Sebta section. Its width varies from a minimum of about $14 \mathrm{Km}$ at Tarifa-Punta Cires section and a maximum of $44 \mathrm{Km}$ at Barbate-Tangier section. The strait is heavily used by shipping and oil transport, being one of the most chronically polluted regions [14]. Notice that this example is only schematic, since the flow conditions and capacities of pollution sources in the strait partially correspond to the real situation. Our main objective in this numerical example is to test the capability of the adaptive SRNH scheme with VanAlbada limiter to handle complex geometry and irregular topography. 

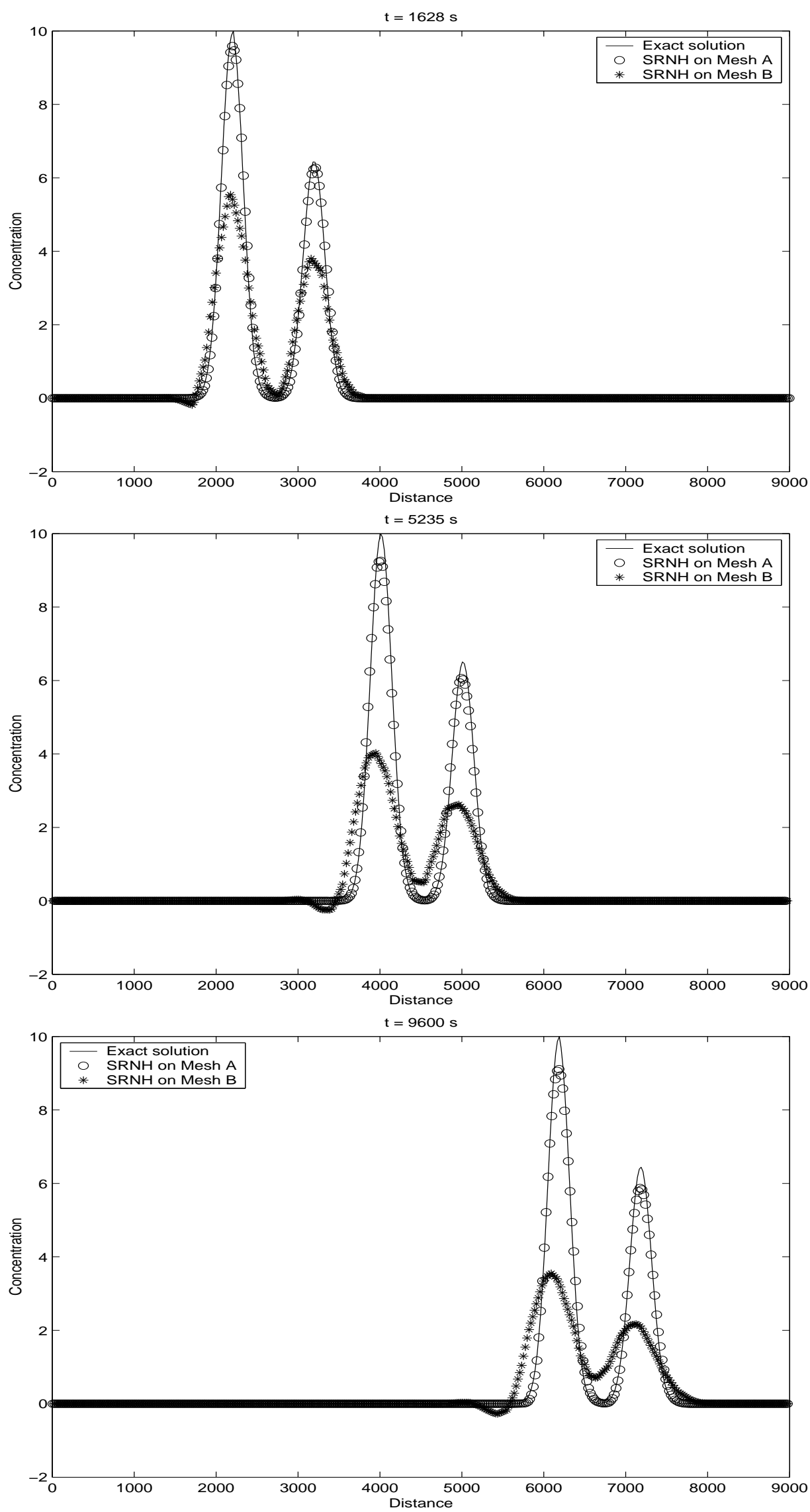

Figure 4.9: Cross sections of the pollutant concentration at the main diagonal $(x=y)$. 

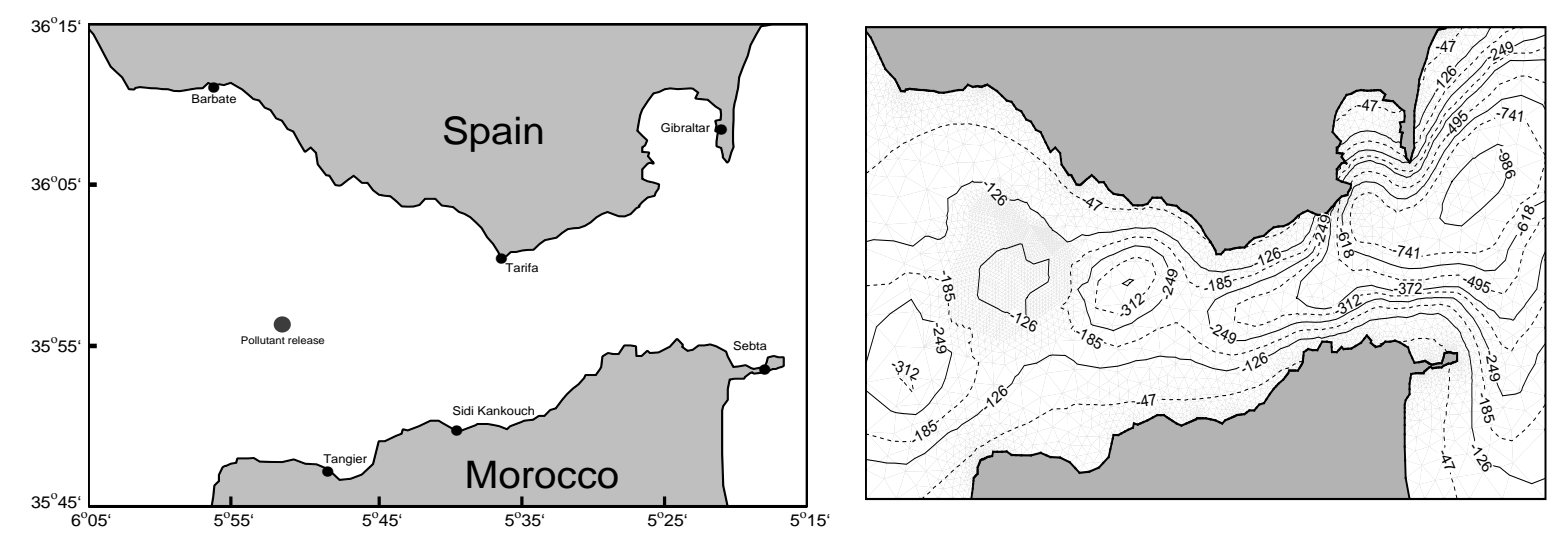

Figure 4.10: Definition of the strait of Gibraltar (left) and its bathymetry (right).

Our problem statement consists of solving the equations (2.6) in the spatial domain shown in Figure 4.10 subject to a pollution release at the inlet of the strait exactly in the mid-axis Barbate-Tangier. We assume a constant diffusion coefficient $D_{x x}=D_{y y}=0.001 \mathrm{~m}^{2} / \mathrm{s}$ and $D_{x y}=$ $D_{y x}=0 \mathrm{~m}^{2} / \mathrm{s}$ for the pollutant transport. We also assume that the concentration vanishes at solid boundaries and we apply zero pollutant flux at the other boundaries. The bathymerty of the strait is very irregular with different spatial scales which needs careful treatment and may causes sever numerical difficulties. In our computations, we used the bathymetry shown in the right plot of Figure 4.10. Similar bathymetry has been used for many numerical studies on mean flow in the strait of Gibraltar, see $[23,6,16]$ among others. According to González et al. [16], the bottom friction Manning coefficient is set to $0.001 \mathrm{~s} / \mathrm{m}^{1 / 3}$ and the problem is forced with the main semidiurnal $M_{2}$ tide with a $25 \mathrm{~cm}$ amplitude which produces a maximum current of approximately $1 \mathrm{~m} / \mathrm{s}$ through the strait inlet and also corresponds to the annual mean of the Atlantic input flux [6]. Hence, the model is equipped at the open boundaries with boundary conditions that simulate the main tidal component $M_{2}$. It should be pointed out that values of the topography were calibrated to cover all the unstructured meshes used at each level of refinement. Certainly, this will add some computational effort to the adaptive SRNH algorithm.

In Figure 4.11 we present the computed results for the adaptive SRNH scheme with VanAlbada limiter at four different times $t=1,2,3$ and 4.5 hours corresponding to the time required by the pollutant to exit the strait if no wind effects are taken into account. In this figure we show the adapted meshes, velocity fields and contours of the normalized concentration, $C / C_{\max }$, where $C_{\max }$ denotes the maximal value of the initial released concentration. It is clear that the adaptive mesh procedure was able to capture the complex features of the flow with a high level of accuracy. The results also show that the adaptive SRNH scheme with VanAlbada limiter was able to predict complex wave interactions with accuracy and to capture pollutant concentration with sharp resolution. By using an adaptive grid, high resolution is automatically obtained in those regions where the pollutant concentration gradients are steep such as the moving fronts. For the conditions considered, the adaptive SRNH scheme with VanAlbada limiter gives a shock-capturing method with very little numerical dissipation, even after long time simulations are carried out.

Since physical diffusion is incorporated into the pollutant transport equation, the maximum concentration was reduced to about $14 \%$ and $50 \%$ after 3 hours and 4.5 hours, respectively. However, for the SRNH scheme with MinMod limiter the maximum concentration was reduced to about $6 \%$ and $21 \%$ after 3 hours and 4.5 hours, respectively. In addition, as in the pure advection tests, the SRNH scheme on fixed meshes exhibits negative concentrations in the vicinity of steep gradients which highly increases as the time progresses during the transport of pollutant. For the case of nonconservative pollutants, the accumulation of these negative concentrations would 

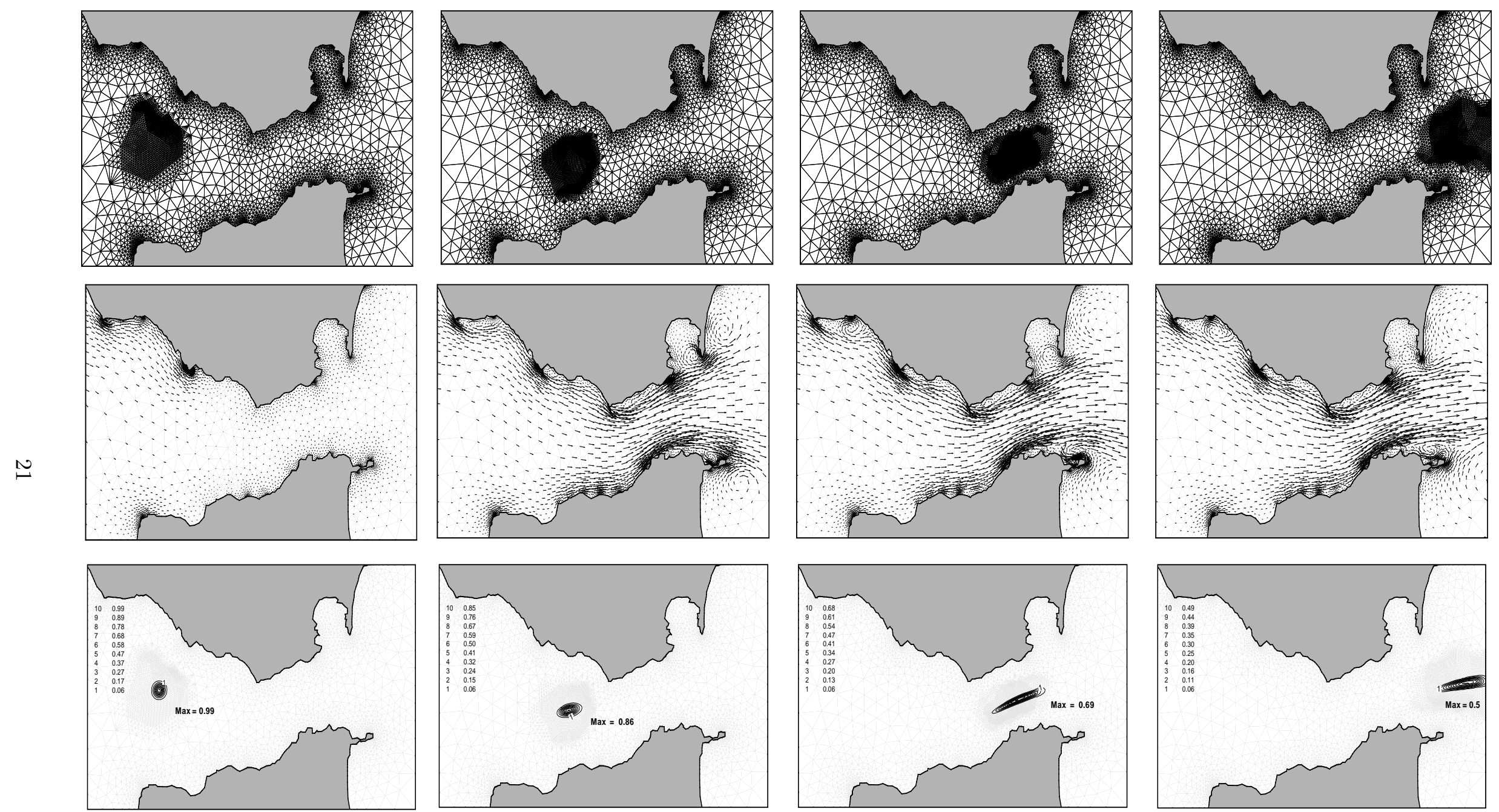

Figure 4.11: Adapted meshes (first row), velocity vectors (second row) and pollutant concentration (third row) at different simulation times. From left to right $t=1,2,3$ and 4.5 hours. 
deteriorate the accuracy of numerical models which may produce physically unrealistic solutions. The SRNH scheme with VanAlbada limiter however, solves such pollution situations accurately.

The CPU times are 5514, 9231 and $11325 s$ for the simulation times $t=2,3$ and 4.5 hours, respectively, on the same Pentium $\mathrm{PC}$ as in the previous test example. It should be noted that the final adaptive grid at $t=4.5$ hour s consists of 9944 elements and 5279 nodes. Therefore by using adaptive meshes, the SRNH scheme with VanAlbada limiter efficiently achieves high resolution in cases where there are locally high gradients. On the other hand, this example shows that large scale shallow water models with irregular topography can be efficiently solved using personal computers without relying on complicated supercomputing resources. It is noteworthy that the computational time necessary to run the tests shown with the SRNH method on fixed meshes was about 7 times the computational time needed to carry out the same tests with SRNH method that used adaptive meshes.

\section{Conclusions}

A simple finite volume non-homogeneous Riemann solver for two-dimensional pollutant transport by shallow water flows has been developed and tested for three examples. The method uses unstructured meshes offering a full adaptation of the grid to the complex geometry by monotiring the pollutant concentration in the computational domain. The method exhibits many desirable properties in the numerical solution of shallow water flows and it is attractive for general purpose flow modelling for several reasons including:

- Accurate prediction of both, the free surface and the scalar transport with correct conservation properties.

- Good convergence behaviors with respect to computational refinement and high efficiency compared with computations on fixed meshes.

- Accurate representation of free surface response to the topography such that all shallow water flow regimes (subcritical, supercritical, etc.) are accurately resolved.

- Implementation on triangular grids that can be easily generated for pollutant transport in complex geometries.

- Ability to handle arbitrary topography in shallow water equations and pure advection in pollutant transport.

The results indicate that this simple approach can be very effective in modeling pollutant transport in rivers and coastal regions. Obviously, it is important to perform mesh adaptation with respect to all flow variables. For example, using the water depth or water surface as monitoring function the resolution in the water flows may be improved. Therefore, our ongoing research focuses on the development of error indicators for both pollutant concentration and hydraulic variables.

Acknowledgment. This work was partly supported by a visiting grant from the Université Paris 13 (France). The authors wish to thank M. González for making the data for the strait of Gibraltar available. 


\section{References}

[1] R. Abgrall, B. Nkonga, and R. Saurel, "Efficient numerical approximation of compressible multi-material flow for unstructured meshes", Comput. Fluids 32, no. 4, 571-605 (2003).

[2] Z. Ahmad, U.C. Kothyari, "Time-line cubic spline interpolation scheme for solution of advection equation", Comput. Fluids. 30, 737-752 (2001).

[3] A.A. Akanbi, N.D. Katopodes, "Models for Flood Propagation on Initially Dry Land", J. Hydraulic Engineering (ASCE). 114, 686-695 (1988).

[4] F. Alcrudo, F. Benkhaldoun, "Exact Solutions to the Riemann Problem of the Shallow Water Equations with a Bottom Step", Comput. Fluids. 30, 643-671 (2001).

[5] F. Alcrudo, P. Garcia-Navarro, "A High Resolution Godunov-type Scheme in Finite Volumes for the 2D Shallow Water Equation", Int. J. Numer. Methods in Fluids. 16, 489-505 (1993).

[6] J.I. Almazán, H. Bryden, T. Kinder, G. Parrilla, eds, "Seminario Sobre la Oceanografía Física del Estrecho de Gibraltar", SECEG, Madrid, (1988).

[7] E. Audusse, F. Bouchut, M.O. Bristeau, R. Klein, B. Perthame, "A Fast and Stable WellBalanced Scheme with Hydrostatic Reconstruction for Shallow Water Flows", SIAM J. Sci. Comp. 25, 2050-2065 (2004).

[8] I. Babuska, T. Strouboulis, C.S. Updhyay, S.K. Gangaraj, "A posteriori estimation and adaptive control of the pollution error in the $h$-version of finte-element method", Int. J. Numer. Methods Eng. 38, 4207-4235 (1995).

[9] F. Benkhaldoun, L. Quivy, "A Non Homogeous Riemann Solver for Shallow Water and Two Phase Flows", Flow Turbulence Combustion. 76 391-402 (2006).

[10] F. Benkhaldoun, "Analysis and Validation of a new Finite Volume Scheme for Nonhomogeneous Systems", Finite Volumes for Complex Applications IV: Problems \& Perspectives, Hermes Science Publications, R. Herbin, D. Kroner Eds. 269-276 (2002).

[11] A. Bermúdez, M.E. Vázquez, "Upwind Methods for Hyperbolic Conservation Laws with Source Terms", Comput. Fluids. 23, 1049-1071 (1994).

[12] I. Elmahi, F. Benkhaldoun, R. Borghi, S. Raghay, "Ignition of fuel issuing from a porous cylinder located adjacent to a heated wall: A numerical study", Combustion Theory and Modelling. 8, 789-809 (2004).

[13] J.M. Greenberg, A.Y. Le Roux, "A Well Balanced Scheme for the Numerical Processing of Source Terms in Hyperbolic Equation", SIAM J. Numer. Anal. 33, 1-16 (1996).

[14] F. Gómez, "The Role of the Exchanges through the Starit of Gibraltar on the Budget of Elements in Western Mediterranean Sea: Consequences of Humain-Induced Modifications", Marine Pollution Bulletin. 46, 685-694 (2003).

[15] M. González, M. Seaïd: "Finite Element Modified Method of Characteristics for Shallow Water Flows: Application to the Strait of Gibraltar". Progress in Industrial Mathematics. 8, 518-522 (2005).

[16] M. González, A. Sánchez-Arcilla, "Un Modelo Numérico en Elementos Finitos para la Corriente Inducida por la Marea. Aplicaciones al Estrecho de Gibraltar", Revista Internacional de Métodos Numéricos para Cálculo y Diseño en Ingeniería. 11, 383-400 (1995). 
[17] M. Heniche, Y. Secretan, P. Boudreau, M. Leclerc, "A Two-Dimensional Finite Element Drying-Wetting Shallow Water Model for Rivers and Estuaries", Advances in Water Resources. 23, 359-372 (2000).

[18] F. Hermeline, "Une méthode automatique de maillage en dimension n", PhD thesis, Université de Paris 6. (1980).

[19] M.S. Horrit, "Development of Physically Based Neshes for Two-Dimensional Models of Meandering Channel flow", Int. J. Numer. Methods in Engineering. 47, 2019-2037 (2000).

[20] I.D. James, "Modelling Pollution Dispersion, the Ecosystem and Water Quality in Costal Waters: A Review", Environ. Model Software. 17, 363-385 (2002).

[21] T. Komatsu, K. Ohgushi, K. Asai, "Refined numerical scheme for advective transport in diffusion simulation", J. Hydraulic Eng. 123, 41-50 (1997).

[22] A. Kurganov, D. Levy, "Central-Upwind Schemes for the Saint-Venant System", Math. Model. Numer. Anal. 36, 397-425 (2002).

[23] J.G. Lafuente, J.L. Almazán, F. Catillejo, A. Khribeche, A. Hakimi, "Sea level in the strait of Gibraltar: Tides", Int. Hydrogr. Rev. LXVII. 1, 111-130 (1990).

[24] R.J. Leveque, "Balancing Source Terms and Flux Gradients in High-Resolution Godunov Methods: The Quasi-steady Wave-Propagation Algorithm", J. Comp. Physics. 146, 346-365 (1998).

[25] B. Lin, R.A. Falconer, "Tidal flow and transport modeling using ultimate quickest scheme", J. Hydraulic Eng. 123, 303-314 (1997).

[26] J.M. Masella, I. Faille, T. Gallouët, "On an Approximate Godunov Scheme", Int. J. Comp. Fluids Dynamics. 13, 133-149 (1999).

[27] T. Ming-Hen, "The Improved Surface Gradient Method for Flows Simulation in Variable Bed Topography Channel using TVD-MacCormack Scheme", Int. J. Numer. Methods in Fluids. 43, 71-91 (2003).

[28] K. Mohamed, "Simulation numérique des systèmes non homogènes", PhD. thesis, Université de Paris 13, (2005).

[29] L.A. Monthe, F. Benkhaldoun, I. Elmahi, "Positivity preserving finite volume Roe schemes for transport-diffusion equations", Comput. Methods Appl. Mech. Engrg. 178, 215-232 (1999).

[30] B. Perthame, C. Simeoni, "A Kinetic Scheme for the Saint-Venant System with a Source Term", CALCOLO. 38, 201-231 (2001).

[31] P.L. Roe, "Approximate Riemann Solvers, Parameter Vectors and Difference Schemes", J. Comp. Physics. 43, 357-372 (1981).

[32] S. Sahmim, F. Benkhaldoun, F. Alcrudo, "A Sign Matrix Based Scheme for Quasi-Hyperbolic Non-Homogeneous PDEs with an Analysis of the Convergence Stagnation Problem", Submited to J. Comput. Phys. (2006).

[33] S. Sahmim, F. Benkhaldoun, "Schéma SRNHS: Analyse et Application d'un Schéma aux Volume Finis dédié aux Systémes non Homogénes", ARIMA. 1 1-15 (2006).

[34] M. Seaïd, "Non-oscillatory relaxation methods for the shallow water equations in one and two space dimensions", Int. J. Numer. Methods in Fluids. 46, 457-484 (2004). 
[35] E.F. Toro, "Shock-Capturing Methods for Free-Surface Shallow Waters", Wiley Chichester 2001

[36] M.E. Vázquez, "Improved Treatment of Source Terms in Upwind Schemes for the Shallow Water Equations in Channels with Irregular Geometry", J. Comp. Physics. 148, 497-526 (1999).

[37] S. Vukovic, L. Sopta, "ENO and WENO schemes with the exact conservation property for one-dimensional shallow-water equations", J. Comp. Physics. 179, 593-621 (2002).

[38] Y. Xing, C. Shu, "High Order Well-Balanced Finite Volume WENO Schemes and Discontinuous Galerkin Methods for a Class of Hyperbolic Systems with Source Terms", J. Comp. Physics. 214, 567-598 (2006).

[39] J.G. Zhou, D.M. Causon, C.G. Mingham, D.M. Ingram, "The Surface Gradient Method for the Treatment of Source Terms in the Shallow Water Equations", J. Comp. Physics. 20, 1-25 (2001). 